\title{
Universiteit
}

Leiden

The Netherlands

\section{Block copolymers confined in a nanopore: Pathfinding in a curving and frustrating flatland}

Sevink, G.J.A.; Zvelindovsky, A.V.

\section{Citation}

Sevink, G. J. A., \& Zvelindovsky, A. V. (2008). Block copolymers confined in a nanopore:

Pathfinding in a curving and frustrating flatland. Journal Of Chemical Physics, 128(8), 084901. doi:10.1063/1.2829406

Version: $\quad$ Not Applicable (or Unknown)

License: $\quad$ Leiden University Non-exclusive license

Downloaded from: https://hdl.handle.net/1887/62392

Note: To cite this publication please use the final published version (if applicable). 


\section{Block copolymers confined in a nanopore: Pathfinding in a curving and frustrating flatland}

G. J. A. Sevink, and A. V. Zvelindovsky

Citation: The Journal of Chemical Physics 128, 084901 (2008);

View online: https://doi.org/10.1063/1.2829406

View Table of Contents: http://aip.scitation.org/toc/jcp/128/8

Published by the American Institute of Physics

\section{Articles you may be interested in}

Self-assembly of diblock copolymers confined in cylindrical nanopores

The Journal of Chemical Physics 127, 114906 (2007); 10.1063/1.2768920

Effect of surface field on the morphology of a symmetric diblock copolymer under cylindrical confinement

The Journal of Chemical Physics 124, 104906 (2006); 10.1063/1.2178802

Morphology of symmetric block copolymer in a cylindrical pore

The Journal of Chemical Physics 115, 8226 (2001); 10.1063/1.1403437

Dynamic simulation of diblock copolymer microphase separation

The Journal of Chemical Physics 108, 8713 (1998); 10.1063/1.476300

Particle size-induced transition between surface segregation and bulk aggregation in a thin film of athermal polymer-nanoparticle blends

The Journal of Chemical Physics 146, 014904 (2017); 10.1063/1.4973608

Symmetric diblock copolymers in nanopores: Monte Carlo simulations and strong-stretching theory

The Journal of Chemical Physics 126, 024903 (2007); 10.1063/1.2406078

\section{AIP | the Joumal of Chemical Physics}

PERSPECTIVES 


\title{
Block copolymers confined in a nanopore: Pathfinding in a curving and frustrating flatland
}

\author{
G. J. A. Sevink ${ }^{\mathrm{a})}$ \\ Leiden Institute of Chemistry, Leiden University, P.O. Box 9502, 2300 RA Leiden, The Netherlands \\ A. V. Zvelindovsky \\ Centre for Materials Science, University of Central Lancashire, Preston PRI 2HE, United Kingdom
}

(Received 27 April 2007; accepted 5 December 2007; published online 25 February 2008)

\begin{abstract}
We have studied structure formation in a confined block copolymer melt by means of dynamic density functional theory. The confinement is two dimensional, and the confined geometry is that of a cylindrical nanopore. Although the results of this study are general, our coarse-grained molecular model is inspired by an experimental lamella-forming polysterene-polybutadiene diblock copolymer system [K. Shin et al., Science 306, 76 (2004)], in which an exotic toroidal structure was observed upon confinement in alumina nanopores. Our computational study shows that a zoo of exotic structures can be formed, although the majority, including the catenoid, helix, and double helix that were also found in Monte Carlo nanopore studies, are metastable states. We introduce a general classification scheme and consider the role of kinetics and elongational pressure on stability and formation pathway of both equilibrium and metastable structures in detail. We find that helicity and threefold connections mediate structural transitions on a larger scale. Moreover, by matching the remaining parameter in our mesoscopic method, the Flory-Huggins parameter $\chi$, to the experimental system, we obtain a structure that resembles the experimental toroidal structure in great detail. Here, the most important factor seems to be the roughness of the pore, i.e., small variations of the pore radius on a scale that is larger than the characteristic size in the system. (c) 2008 American Institute of Physics. [DOI: 10.1063/1.2829406]
\end{abstract}

\section{INTRODUCTION}

Pattern formation of block copolymers in constraint situations or confinement is an important topic in polymer research, since the meso-or microstructure can be much better controlled when compared to the bulk. In general, molecular conformations and assembly are strongly influenced by confinement. In the absence of external constraints, the microstructure is dictated by the interaction between segments comprising the copolymer, the volume fraction of the blocks, and the molecular architecture. In a confined system, however, interfacial interactions, symmetry breaking, structural frustration, and confinement-induced entropy loss play a determining role and may lead to structures that differ from the ones found in bulk. As a direct result phase separation in confinement has been the subject of extensive theoretical and experimental studies. ${ }^{1}$ The intriguing prospects from a technological viewpoint are the novel structures that can be achieved and that may serve as scaffolds for other nanostructures.

The simplest example of a block copolymer is a linear $A B$ diblock copolymer. In the bulk several stable periodic microstructures can be formed, among which are lamellar, hexagonal, and body-centered cubic phases. ${ }^{2}$ The equilibrium behavior for $A B$ diblock copolymers in the bulk has been mapped out both experimentally and theoretically; the situation for more complex multiblock and/or branched

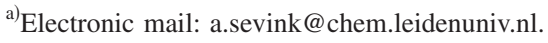

block copolymers is much less clear. Here, we focus on symmetric or nearly symmetric $A B$ diblock copolymers. In the bulk, these copolymers microphase separate into lamellar microdomains with a characteristic equilibrium period $L_{0}$, where grains of ordered lamellar microdomains are randomly oriented. Global orientation of the microdomains can be induced by confinement of the block copolymer in thin supported films or slits (one-dimensional confinement). ${ }^{3-6}$ Additionally, the interplay between surface fields (interaction with confining surfaces) and confinement effects (commensurability) can affect the phase behavior, and lead to the formation of surface reconstructions or hybrid structures. ${ }^{7-9}$ Both statics and dynamics ${ }^{10,11}$ of thin film phase behavior have been well studied.

An obvious next step in experimental and theoretical research is to consider systems where the confinement is effectively two dimensional. This is the case when the melt is confined inside a cylindrical nanopore of radius $R$. From a conceptual point of view, this type of confinement differs from the one-dimensional (1D) confinement since (1) there is only one confining surface, so there is only one surface field; (2) the nanopore solid surface is curved. By definition, surface reconstructions (analogs of the uni-or multilayer parallel lamellae $L_{\|}$in slits) are therefore curved as well, which has an effect on the entropy contribution of the structure in the free energy. This can either result in a breakup of the nanopore in two relatively independent regions (close and away from the pore surface), or in curved multilayer structures that experience lamellar bending throughout the pore, with an 
increased bending towards the center of the pore, (3) just like in slits, surface reconstructions may be able to adapt their layer spacing to some extent in order to suit the cylinder radius $R$. One can expect the influence of this type of frustration to be less than in slits, as there is no block preference in the center of the pore. However, for small $R$, structures can experience substantial frustration due to incommensurability. To resolve this unfavorable situation, the lamellae can adapt an orientation perpendicular to the nanopore wall or the chains may find alternative packing and form hybrid structures, like in a slit, and (4) in addition to the commensurability issue for parallel structures or surface reconstructions [see (2)] the length of a nanopore can also affect the formation of perpendicular structures (incommensurability along the pore). In slits, these perpendicular structures are found for strong surface fields at incommensurable film thickness, and for weak surface fields independent of the film thickness. In nanopores, a mismatch of the pore length and the natural spacing $L_{0}$ can lead to extensional forces, and may stabilize hybrid structures as well.

Based on rather small data set of surface interactions and nanopore radii $R$, two pioneering computational studies (by Monte Carlo $^{12}$ and dynamic self-consistent field theory ${ }^{13}$ ) identified two structures: A slab and a multiwall tube morphology. ${ }^{13}$ In analogy with the classification scheme for structures in a slit, ${ }^{14,15}$ slabs can be associated with lamellae perpendicular to the confining surface $\left(L_{\perp}\right)$ and multiwall tubes with surface reconstructions: Lamellae that are parallel to the confining surface $\left(L_{\|}\right)$. One should note that this remark implies that the nature of these structures is the same as the lamellar structure found in bulk. It was concluded that two mechanisms control the structure formation in a nanopore: (1) In the case of weak surface interactions the lamellae orient perpendicular to the pore wall to form lamellar slabs and (2) for strong surface interactions, one of the blocks segregates preferentially to the pore wall, and the lamellae line up and bend to form concentric cylinders, the number of which is determined by the cylinder radius. The effect of commensurability was found to be less significant in this type of confinement, since no perpendicular structures were observed for incommensurate $R$ and higher surface interactions.

Very recently, the significance of these theoretical studies increased dramatically by the appearance of a number of intricate experimental studies of polystyrene-block-polybutadiene ${ }^{16-19} \quad$ (PS- $b$-PB) and polystyrene-block-poly(methyl methacrylate) ${ }^{20}$ (PS- $b$-PMMA) diblock copolymers confined in nanopores. In these studies, bulk lamella-, cylinder-, and sphere-forming block copolymers were introduced into nanoscopic cylindrical pores in alumina membranes. For bulk lamella-forming block copolymers the predicted morphology ${ }^{12,13}$ of slabs was not found, most probably due to the strong disbalance of surface energies in the experimental setup (although these were not measured as such). The most frequent found structure was a concentric cylinder (multiwall tube ${ }^{13}$ ) morphology. However, also a new structure, a stacked-disk or toroidal-type ${ }^{16}$ structure, was found inside a nanopore with an incommensurate pore diameter $\left(d / L_{0} \sim 2.6\right.$, with $d$ the pore diameter).

As a result of these experimental findings, computational studies have considered the phase behavior of confined symmetric diblock copolymers in more detail, by means of selfconsistent field theory ${ }^{21}$ (SCFT) and Monte Carlo ${ }^{22-25}$ (MC) methods. Based on two-dimensional (2D) calculations, the work of Li et al. ${ }^{21}$ aimed at constructing phase diagrams $(\chi N$ vs $f=f_{A}$ ), and found lamellae for $f_{A}=1 / 2$ (the diagram was calculated for a single radius $R=8.5 R_{g}$ ). Although this work can be seen as a stepping stone for the understanding of the nature of phase transitions under varying conditions, their findings do not directly relate to the essentially threedimensional (3D) structures found in experiments. For instance, the slab morphology is out of the scope of their calculations. Moreover, the influence of the nanopore radius and the surface energetics was not considered in detail. Based on lattice MC simulations, Chen et al.; Feng and Ruckenstein, and Wang indeed found several new structures in three dimensions: A single helix, catenoid cylinder, gyroidal, stacked circle, and disordered structure in Ref. 22; a mesh, lamellae parallel to the pore axis, single helix, and double helix structure in Ref. 23; and catenoid cylinder in Ref. 25. A later study of Feng and Ruckenstein concentrated on the stability of the helical structure. ${ }^{24}$ From a visual comparison we conclude that the mesh structure ${ }^{23}$ is the same as catenoid cylinder. ${ }^{22}$ Similarly, one can claim that the gyroidal structure is, in fact, a defected structure, and comprises a coexistence of a mesh and a double helix structure. ${ }^{22}$ We adapt a common notation, and conclude that this reduces the number of computed nanopore structures to seven: Stacked disk, concentric cylinder, lamellae parallel to the pore axis, catenoid cylinder, disordered, single helix, and double helix.

Although these new structures were found, a fundamental understanding of the underlying mechanisms such as achieved for thin films (effective 1D confinement) is absent, except for the MC study of Wang. ${ }^{25}$ However, the latter study is restricted to strong segregation, and most remarkably several of the other structures (for instance, the interesting helix and double helix) were not found at all. Moreover, the origin of the experimental stacked-disk or toroidal-type ${ }^{16}$ structure is still unexplained. More fundamental studies, including scans of a larger parameter space, are clearly needed for a deeper understanding.

In principle, one can anticipate several regimes. Equilibrium morphologies are minima of the free energy, containing both energetic and entropic contributions. In the absence of any surface field and for a strong surface field, either one of these contributions is dominating, leading to the stacked-disk and concentric cylindrical structures. Between these regions, for weak surface fields, the chains have more flexibility to adapt their packing and the system can and apparently will adapt other morphologies. Incommensurability may play a subtle role here. The details of this interplay remain to be determined, for instance, by the calculation of a structure diagram in three dimensions, depending on $R$ and the surface interaction strength. Since we consider lamella-forming sys- 
tems, $f_{A}$, the volume fraction of the $A$-block, is fixed (in contrary to Ref. 21) and the diagram is conceptually similar to the one on Ref. 14 for confined films.

Moreover, two factors have not been considered in detail yet: The influence of the kinetic pathway and the value of $\chi_{A B} N$, where $N$ is the total length of the block copolymer. Although we will not focus into detail in the latter factor, from Ref. 21 one can conclude that an increase of $\chi_{A B} N$ may lead to a decrease of the number of concentric lamellae. Here, we focus on calculating the structural diagram and the question of stability. Whether interesting structures such as double helix can actually be manufactured experimentally may be a very subtle issue. It is known that in strong confinement the dynamics may slow down and structures may be frozen into metastable states. This is particularly the case when the free energy difference between states is small or when there is a large energy barrier between different local minima of the free energy. In nanopores, this situation was actually observed in MC studies: In Ref. 22 for weak surface fields up to three different structures were found. Previously, a dynamic density functional theory (DDFT) study ${ }^{13}$ identified the single helix structure as a long-living intermediate structure between complete mixing and stacked disks for zero surface field. We will use this method here to consider the experimental system of Ref. 16 in detail. In contrast to traditional schemes of polymer phase separation dynamics where a Landau Hamiltonian is used with vertex functions calculated following the random phase approximation (see, e.g., Ref. 26), we numerically calculate the free energy $F$ of polymer system consisting of Gaussian chains in a mean field environment using a path integral formalism. ${ }^{27,28}$ Our approach uses essentially the same free energy functional as in SCF calculations of equilibrium block copolymer morphologies by Matsen and Schick, ${ }^{29}$ but complements the static SCF calculations by providing a dynamical picture of the system.

\section{METHOD}

Here, we shortly discuss the DDFT method ${ }^{27,30}$ for a bulk lamella-forming diblock copolymer melt. The diblock copolymers are modeled by a $A_{N_{A}} B_{N_{B}}$ Gaussian chain $(N$ $\left.=N_{A}+N_{B}, f_{I}=N_{I} / N\right)$. The confined geometry is a cylindrical pore with varying diameter $R$. Periodic boundary conditions apply in the $x$-direction, along the pore. Calculations are carried out on a cubic $L_{x} \times L_{y} \times L_{z}$ grid with a spacing $\Delta x$ that is related to the Gaussian bond length $a$ via $a \Delta x^{-1}=1.1543$. $^{31}$ The spacing $\Delta x$ is equal among different pore systems, making the free energy per volume element of $\Delta x^{3}$ easily comparable. Unless mentioned otherwise, all spacings are in units of this basic variable $\Delta x$. The pore is introduced into the simulation volume $V$ by a masking technique; as a result the simulation volume contains both pore and mask points. ${ }^{30}$ In mask points, elements of the subset $V^{0}=\{\mathbf{r}=(x, y, z)$ $\left.\in V \mid\left\|(x, y, z)-\left(x, y_{0}, z_{0}\right)\right\|>R\right\}$ with $y_{0}=L_{y} / 2, z_{0}=L_{z} / 2$, all concentration and external potential field values are set to zero, except for the auxiliary field $\rho_{M}(\mathbf{r})=1$ that represents the mask itself. ${ }^{30}$ The free energy for unconfined systems is given by (see Ref. 27 for details)

$$
\begin{aligned}
F[\rho]= & -k T \ln \frac{\Phi^{n}}{n !}-\sum_{I} \int_{V} U_{I}(\mathbf{r}) \rho_{I}(\mathbf{r}) d \mathbf{r} \\
& +\frac{1}{2} \sum_{I, J} \int_{V^{2}} \varepsilon_{I J}\left(\left|\mathbf{r}-\mathbf{r}^{\prime}\right|\right) \rho_{I}(\mathbf{r}) \rho_{J}\left(\mathbf{r}^{\prime}\right) d \mathbf{r} d \mathbf{r}^{\prime} \\
& +\frac{\kappa}{2} \int_{V}\left(\sum_{I} \nu\left(\rho_{I}(\mathbf{r})-\rho_{I}^{0}\right)\right)^{2} d \mathbf{r} .
\end{aligned}
$$

Here, $k$ is the Boltzmann constant, $T$ is the temperature, $n$ is the number of polymer molecules in the volume $V$ occupied by the system, and $\Phi$ is the intramolecular partition function for ideal polymer chains. The parameter $\kappa$ determines the compressibility of the system (the dimensionless $\kappa^{\prime}=\beta \kappa \nu$ $=20.65$, with $\nu$ the bead volume), and $\rho_{I}^{0}$ is the mean concentration of the $I$-block (where the average is taken over $\left.V \backslash V^{0}\right)$. For $\kappa \rightarrow \infty$ the system becomes incompressible. The external potentials $U_{I}$ and the concentration fields $\rho_{I}$ are related via the density functional. ${ }^{28}$ The interchain interactions are incorporated via a mean field with interaction strength controlled by the Flory-Huggins parameters $\chi_{I J}$. In line with our earlier work the interactions are specified by the parameters $\varepsilon_{I J}^{0}$ (in $\mathrm{kJ} / \mathrm{mol}$ ), ${ }^{27,28}$ which are directly related to the Flory-Huggins parameters by $\chi_{I J}=1000 \varepsilon_{I J}^{0} / n_{A} k T$ (with $n_{A}$ Avogadro's number and $T=300$ the temperature in Kelvin). In case of nonzero surface interactions an extra cohesive term is added to the free energy (1) equal to ${ }^{30}$

$$
F^{\text {wall }}=\sum_{I} \int_{V^{2}} \varepsilon_{I M}\left(\left|\mathbf{r}-\mathbf{r}^{\prime}\right|\right) \rho_{I}(\mathbf{r}) \rho_{M}\left(\mathbf{r}^{\prime}\right) d \mathbf{r} d \mathbf{r}^{\prime} .
$$

In line with our earlier work, the interaction kernel is chosen Gaussian, and the important parameter $\epsilon_{I M}^{0}$ denotes the scalar interaction $\operatorname{strength}^{27}$ of bead $I$ with the pore boundary. In the computations, $\varepsilon_{B M}^{0}=0$ indicating that the $B$-blocks have no interaction with the wall, which is appropriate due to the fact that for an incompressible system of diblocks only the effective surface interaction $\xi=\varepsilon_{A M}^{0}-\varepsilon_{B M}^{0}$ is of importance. Using this we obtain

$$
F^{\text {wall }}=\sum_{I} \varepsilon_{I M}^{0} \mathcal{F}\left[\rho_{I}, \rho_{M}\right]=\xi \mathcal{F}\left[\rho_{A}, \rho_{M}\right],
$$

where $\mathcal{F}$ is

$$
\mathcal{F}=\mathcal{C} \int_{V^{2}} e^{-3 / 2 a^{2}\left(r-r^{\prime}\right)^{2}} \rho_{A}(\mathbf{r}) \rho_{M}\left(\mathbf{r}^{\prime}\right) d \mathbf{r} d \mathbf{r}^{\prime}
$$

with $\mathcal{C}=\left(3 / 2 \pi a^{2}\right)^{3 / 2}$ a normalization constant and $a$ the Gaussian bond length. Since the bead-bead interaction $\varepsilon_{A B}^{0}$ is mostly considered a constant, we will use the notation $(R, \xi)$ to denote points in the structural diagram.

For the simplest model, the evolution of the density fields is given by a Langevin equation ${ }^{32}$

$$
\frac{d \rho_{I}(\mathbf{r})}{d t}=M \Delta \mu_{I}(\mathbf{r})+\eta_{I}(\mathbf{r})
$$

with $M$ a constant mobility, $\Delta$ the Laplace operator, and $\eta$ noise, distributed according to the fluctuation-dissipation theorem. Other transport coefficients, like the one for collective Rouse dynamics or reptation, exist, ${ }^{32}$ but are in general 
too computationally demanding. We have recently found that our relatively simple model (5) can be appropriate to describe the experimental dynamics in detail. In Ref. 11 the experimental and calculated dynamics of cylinder- and sphere-forming diblock copolymers under an external electric field was compared, and good agreement was found based on DDFT with constant transport coefficients. In Ref. 10 the calculated dynamics of DDFT was shown to agree in full detail with scanning force microscopy (SFM) measurements of experimental dynamics in a thin film of a concentrated polystyrene-block-polybutadiene-block-polystyrene (SBS) solution. Finally, apart from the extra term in the free energy, confinement is accounted for by the boundary condition for the dynamic equations $n \cdot \nabla \mu_{I}=0$, with $n$ the normal pointing into the solid object. ${ }^{30}$

\section{RESULTS AND DISCUSSION}

\section{A. Nanopores: Data from literature}

Since the nanopore simulation data in literature are rather scattered, ${ }^{12,13,22,23}$ we first present a short overview. A direct comparison of these different studies is complicated by the fact that important system parameters are different. A MC method was used to simulate the phase behavior for a $A_{5} B_{5}$ diblock copolymer ${ }^{23}$ with $\varepsilon_{A B}=0.3,1.0$, and $1.1 \mathrm{kT}$, and for a $A_{10} B_{10}$ diblock copolymer ${ }^{12,22}$ with $\varepsilon_{A B}=1.0 \mathrm{kT}$. In both studies all other interactions are zero: Only the $\varepsilon_{A S}$ (in kT), the interaction between $A$-blocks and the surface, is varied. In the DDFT calculations of Ref. 13, a $A_{8} B_{8}$ system was considered for $\varepsilon_{A B}=2.5 \mathrm{~kJ} / \mathrm{mol}$ and varying $\varepsilon_{A S}$ (in $\mathrm{kJ} / \mathrm{mol}$ ) (in that article, $S$ is actually called $M$ or mask). Using the formula in Sec. II for $T=300 \mathrm{~K}$, we obtain $\chi=1.0$ and $\chi N$ $=16$ (weak segregation). Using the expression from Ref. 33, $\chi \approx 5(\varepsilon / k T)$, we can recalculate the parameters used in the MC studies: $\chi N \approx 15,50$, and 55 (weak to intermediate segregation) for Ref. 23 and $\chi N \approx 100$ in Refs. 12 and 22 (strong segregation), where it should be noted that Ref. 23 concentrates on $\chi N \approx 15$ (weak segregation). As a consequence, the results are distributed between weak, intermediate, and strong segregation regimes. In Fig. 1 we have combined the existing knowledge in schematic diagrams for different $\chi N$; we have only differentiated between stacked disks, concentric cylinders, and alternative structures. It should be noted that the diagram for DDFT is essentially no phase diagrams, as the final structures are pathway dependent, and not minima of the free energy per se. This is a fundamental difference between static calculations aimed at deriving equilibrium morphologies, and our dynamic simulations aimed at mimicking experimental pathways, including visits to longliving metastable states. The important dimensionless spatial coordinate is the ratio of the pore diameter $R$ and the lamellar domain distance $L_{0}$; this parameter is on the vertical axis. On the horizontal axis is $\varepsilon_{A S}$ (in kT) of MC. Since the diblock is symmetric we can restrict ourselves to only positive values. We have used $\varepsilon_{A S}($ in $\mathrm{kT}) \approx 1 / 2 \varepsilon_{A S}^{0}$ (in $\mathrm{kJ} / \mathrm{mol}$ ) for the conversion of the DDFT to the MC value.

We see that concentric cylindrical structures dominate all diagrams when $\varepsilon_{A S}$ is large, irrespective of the value of $\chi N$. In this case, the surface field dominates and one block is
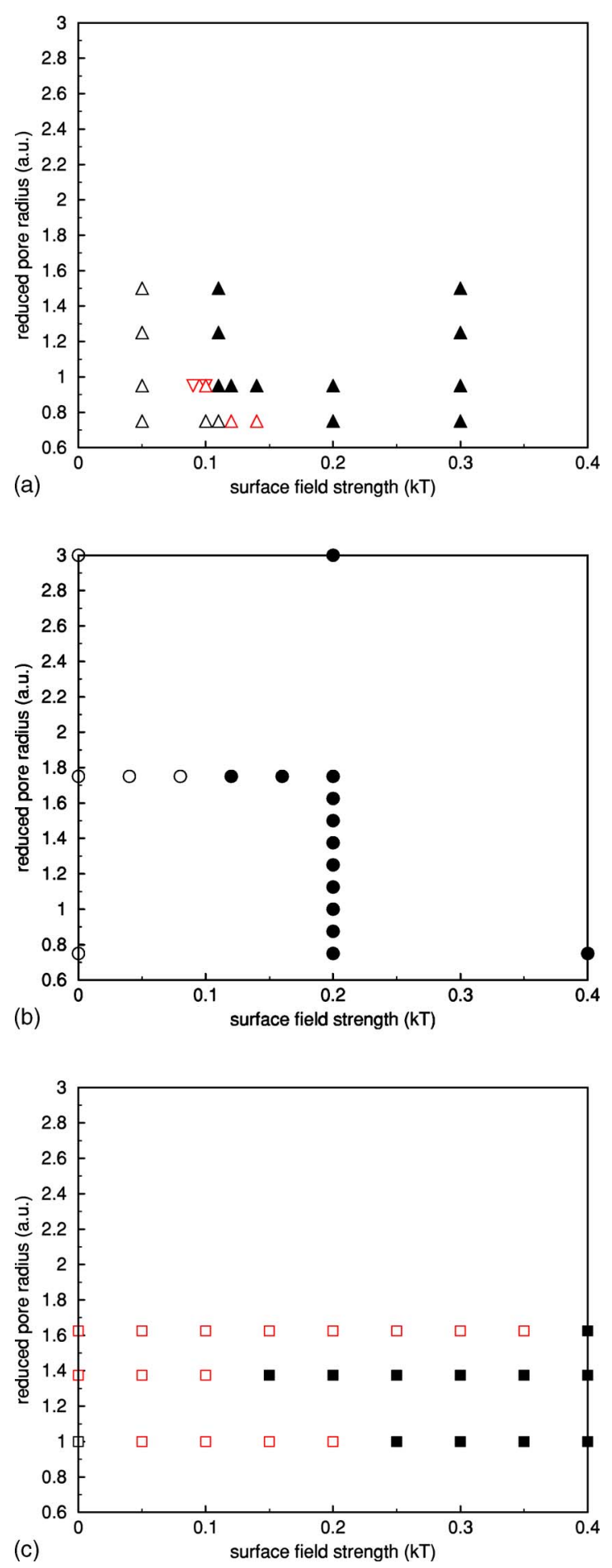

FIG. 1. (Color online) Structural diagram with existing simulation results. Different symbols denote systems in different segregation regimes: (triangles up) a $A_{5} B_{5}$ system with $N \chi \approx 15$, (triangles down) the same system with $N \chi \approx 50$ (Ref. 23), (circles) a $A_{8} B_{8}$ system with $N \chi=16$ (Ref. 13), and (squares) a $A_{10} B_{10}$ system with $N \chi \approx 100$ (Ref. 22). Open symbols represent stacked disks, closed symbols concentric cylinders, and red symbols alternative structures. We have scaled the axes to make a comparison: The vertical axis shows the reduced pore radius $R / L_{0}$, with $R$ the pore diameter and $L_{0}$ the particular lamellar spacing in bulk, the horizontal axis $|\varepsilon|$, and the energetic interactions of $A$ with the surface in the MC method. We have used the relation $\chi \approx 5(\varepsilon / k T)$ (Ref. 33) for the conversion of our energetic FH parameter.

energetically favored at the pore wall. For neutral pores, i.e., in the absence of a surface field $\left(\varepsilon_{A S}=0\right)$, stacked disks dominate. In stacked disks the chains can adopt a packing 
that is similar to the one in normal bulk lamellae. However, for the strongest segregated system stacked disk coexists with a single helix for this surface field. For small nonzero values of $\varepsilon_{A S}$ also other structures can be found.

Although the data are sparse, one can conclude that structures with alternative packing dominate the diagram for large $\chi N$ and relatively small $\varepsilon_{A S}$. Incommensurability cannot be the only important factor since these alternative structures are even found for commensurate pore radii $R / L_{0}=1,{ }^{22}$ although there is some uncertainty since the bulk domain distance $L_{0}$ was not determined explicitly in this study. We can only conclude from these data that especially weak surface fields in combination with strong segregation (high $\chi N$ ) lead to structures with alternative packing.

\section{B. Nanopores: System choice and boundary condition}

The system was chosen to model the experimental polystyrene-block-polybutadiene (PS- $b$-PBD) diblock copolymer in Ref. 16, which has a volume fraction of 0.56 for the butadiene block. The molecular model considered here is a $S_{10} B_{12}\left(N_{S}=10, N_{B}=12\right)$ Gaussian chain. The interaction between $S$ and $B$ beads is chosen as $\varepsilon_{S B}=2.0 \mathrm{~kJ} / \mathrm{mol}$ or $\chi$ $=0.8$, and consequently $\chi N=17.6$. A diblock copolymer melt of this molecular composition $f_{B}=0.545$ forms lamellae in bulk, and the bulk lamellar distance was determined as $L_{0}$ $=8.6$ (from here onwards, all distances are in units of $\Delta x$ ). Commensurability issues in the $x$ direction, along the pore axis, may arise as a result of periodic boundary conditions. Since the effects of periodic boundary conditions have been considered in the literature, ${ }^{34,35}$ we refer to these works for a detailed discussion. Here, we only note that the studies mentioned in the previous paragraph have not considered this effect in detail. Only for one data set in Ref. 22, where both helices and stacked disks were found and no transition between the two different structures, it was shown that the number of MC simulations required to first find a helical structure peaks at particular cylinder lengths (our $L_{x}$ ). General conclusions from this study are not easy to make due to the absence of regularity.

\section{Nanopores: Results and discussion}

We have considered pattern formation in a $S_{10} B_{12}$ diblock copolymer melt confined in a nanopore of length $L_{x}=32=3.72 L_{0}$, except for the larger pores where we have used $L_{x}=34=3.95 L_{0}$ and $L_{x}=36=4.19 L_{0}$. In order to give a unifying description for the structural behavior of slightly asymmetric diblock copolymers in nanopores, we first construct and discuss a diagram of simulated structures. Since these structures are the result of dynamic pathways and therefore may be metastable, we consider their stability by an interpolation procedure described below. Consequently, we focus on several factors that are important for structure formation: Two types of incommensurability issues, due to packing frustration along the pore (associated with perpendicular structures) and perpendicular to the pore (associated with parallel structures), and the kinetic pathway, that may lead to arrested structures. In the second part, we discuss these issues in detail, and unravel the complex interplay of these factors and the surface field.

Packing frustrations along the pore, originating from the requirement of periodicity at the two pore boundaries, have not received much attention in the past, except for the unconfined situation. ${ }^{35}$ In this study, it was shown that this frustration, quantized by $L_{x} / L_{0}$, determines the (in)stability of several perpendicular structures. This effect, which can be related to elongational stress or extensional force in an applied shear field, will be discussed in detail for our results.

In the absence of extensional forces, the analogy to similar substrates in thin films suggests that the phase behavior in pores is due to an interplay of two factors: The strength of the surface field and confinement effects. ${ }^{1,14,36}$ For vanishing or weak surface fields, the elastic chain deformation associated with parallel structures will be avoided, and perpendicular structures are favored instead. Due to the selective blocksurface interaction, parallel structures (surface reconstructions) will be promoted for surface fields above a certain threshold field strength. However, the available space in the confined geometry dictates the degree of chain compression or extension required for parallel structures. Large chain frustrations give rise to an entropically unfavorable situation and may even prevent the formation of parallel structures. Alternative (in thin films, perpendicular) structures are then formed instead. In thin films, the surface fields were shown to be additive and affect only the first layers of structure. ${ }^{36}$ Consequently the available space, quantized by $R / L_{0}$ in pores, is important for both factors. The surface field and frustration due to confinement will strongly influence structure formation in small pores. In larger pores, the surface field has a limited range, and possible structure frustration can be distributed over more layers of structure.

We note that the diblock copolymer considered here is slightly asymmetric. As a consequence, the structure diagram is not completely symmetric as well. In the absence of surface interactions, the shortest $(S)$ part of the chain is preferentially found close to the surface due to entropic effects. The value for which energetic and entropic contributions are balanced shifts to a small positive $\xi .{ }^{14}$ Moreover, for parallel structures in the curved geometry, this chain asymmetry leads to curvature effects, since the spontaneous curvature associated with each of the different blocks is slightly different, and asymmetric packing frustrations due to the different $S$ and $B$ domain sizes. In contrast to thin films, the majority component of the center layer in a parallel structure is not prescribed by surface energetics. Packing frustrations are therefore expected to be less significant than in thin films, where in most cases the block next to the surfaces is prescribed by the surface fields. However, the number of domains/layers for each of the blocks can differ. To focus on this effect we adapt two notations: If the majority component in a parallel structure is $S-B-S-B-S$ or $B-S-B-S-B$, on a line perpendicular to the pore axis from wall to wall, we denote the structure as $L_{\|, 1(1 / 2)}$ (for a concentric cylindrical structure, in line with the slit notation ${ }^{14}$ ), and by the order of the majority component in the layers from wall to center, $S B S$ or $B S B$. Note that here the total number of $S$ and $B$ domains differs (2 or 3 ), as well as the composition of the central 


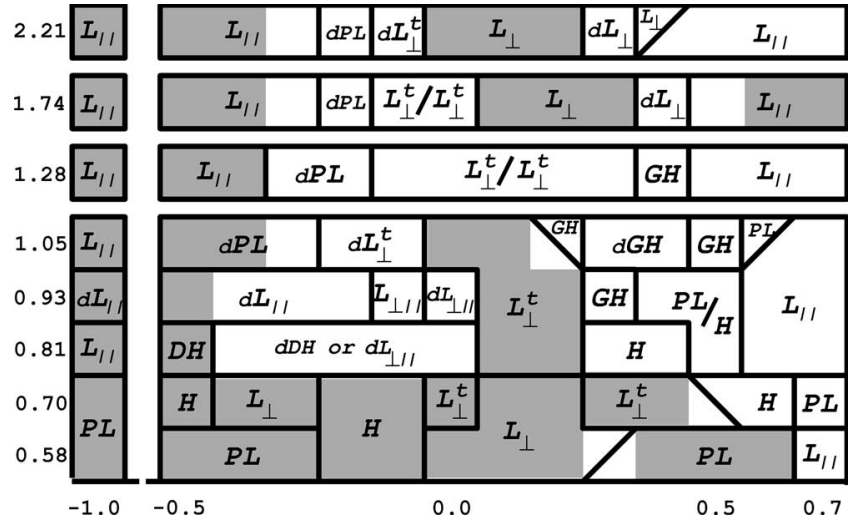

FIG. 2. Nanopore structure diagram for varying pore radius (in $R / L_{0}$ ) and surface field (in $\mathrm{kJ} / \mathrm{mol}$ ). Stable structures for each $R / L_{0}$ (the determination of which is based on the free energies for constant pore length $L_{x}$, see Fig. 5) are denoted by a gray background.

cylinder, associated with the largest curvature. Curvature is an important factor and a complicating factor, when compared to the thin film situation. In principle, each of the layers in a parallel structure can adapt their thickness to some extent in response to global mismatches. Simple volumetric arguments indicate that the domain spacing in nanopore confinement depends on the local curvature, and therefore on the absolute radial position of $S-B$ interfaces in the pore. ${ }^{25}$ This is particular the case in the limit of strong segregation; for weaker segregation, the situation may be different, since the blocks are somewhat miscible. As a result, the spacing may heterogeneously deviate from the bulk domain spacing $\left(L_{0}\right.$ $=8.6$, so $D_{S}=3.9$ and $\left.D_{B}=4.7\right)$, near the pore center, where

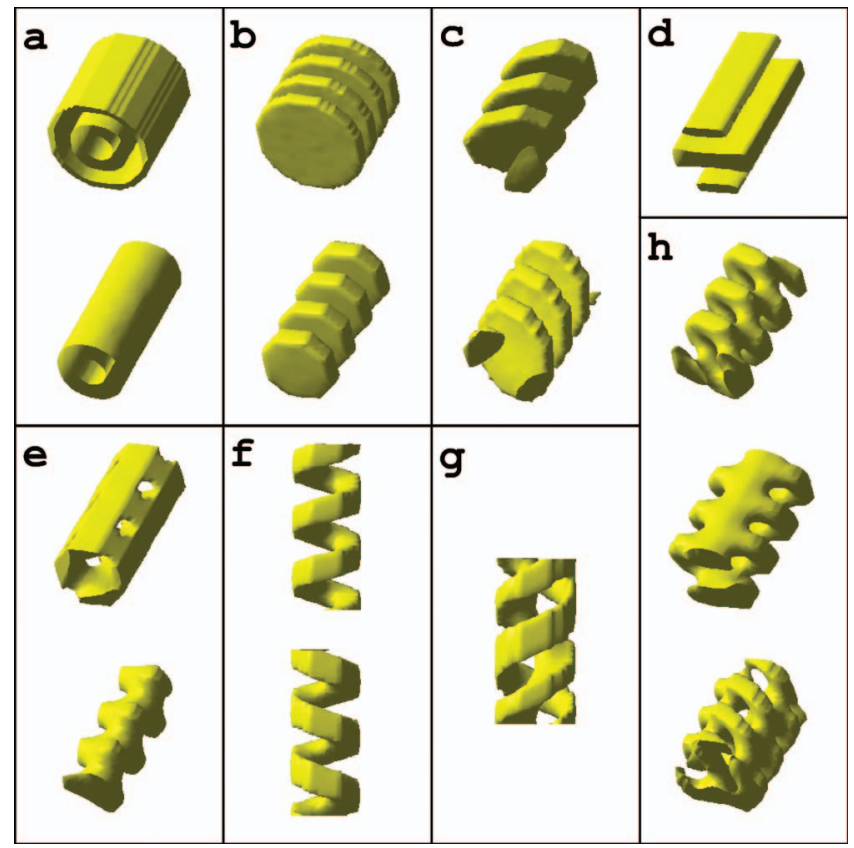

FIG. 3. (Color) Representative 3D nanopore structures for (a) $L_{\|}$, (b) $L_{\perp}$, (c) $L_{\perp}^{\text {tilt }}$, (d) $L_{\perp, \|}$, (e) $P L$, (f) $H$, (g) $D H$, (h) $G H$. The corresponding locations in the structure diagram are: (a) $(1.74,-0.3)$ and $(0.93,0.7)$, (b) $(1.74,0.1)$ and $(0.70,0.2)$, (c) $(0.93,0.1)$ and $(1.05,0.1),(\mathrm{d})(0.93,-0.1),(\mathrm{e})(0.70,-1.0)$ and $(0.70,0.7)$, (f) $(0.70,-0.1)$ and $(0.70,-0.2)$, (g) $(0.81,-0.5)$, and (h) $(0.93,0.3),(1.05,0.5)$, and $(1.28,0.4)$. Isodensity surfaces of the $S$ component for mean value are shown. the curvature is highest, and in the wetting layer, where confinement effects are most severe. For a symmetric diblock copolymer and larger radii $R$, we previously showed that packing frustrations in parallel structures are mainly relieved by rearrangement in the cylindrical center region; in the layered structure away from the center, the chain conformations are rather unaffected by the local curvature. ${ }^{13}$ We will consider this interplay of surface field and frustration in detail for our asymmetric block copolymer.

We have varied the surface interaction in a range of negative and positive values $\xi$, between vanishing (nonselective) to intermediate (selective, $S$ or $B$ ) surface fields. A range of small $R$ up to approximately $L_{0}$ was chosen to consider the details of this interplay in strong confinement. Moreover, also a few larger $R$ were considered to study these effects separately. The structures are displayed in Figs. 2-4, and the free energies associated with these structures in Fig. 5 . In the selected region in the $(R, \xi)$ diagram, we expect that confinement effects, surface fields, and/or the interplay between these two give rise to alternative structures. In the remainder, we use the relevant dimensionless parameter $R / L_{0}$ instead of the bare radius $R$. For all calculated final structures, the free energy was monitored and remained constant.

We introduce a short-hand notation for the perfect structures: Catenoid cylinder is denoted by $P L$ or $P L(I)$ [depending on the sign of $\xi$, the majority component $I$ of the structure is either $S$ (negative) or $B$ (positive)], disordered by $D$, single helix by $H$, and double helix by $D H$. The lamellae parallel to the cylinder axis are perpendicular to the pore wall but also parallel, and we denote them as $L_{\perp, \|}$. In some cases,

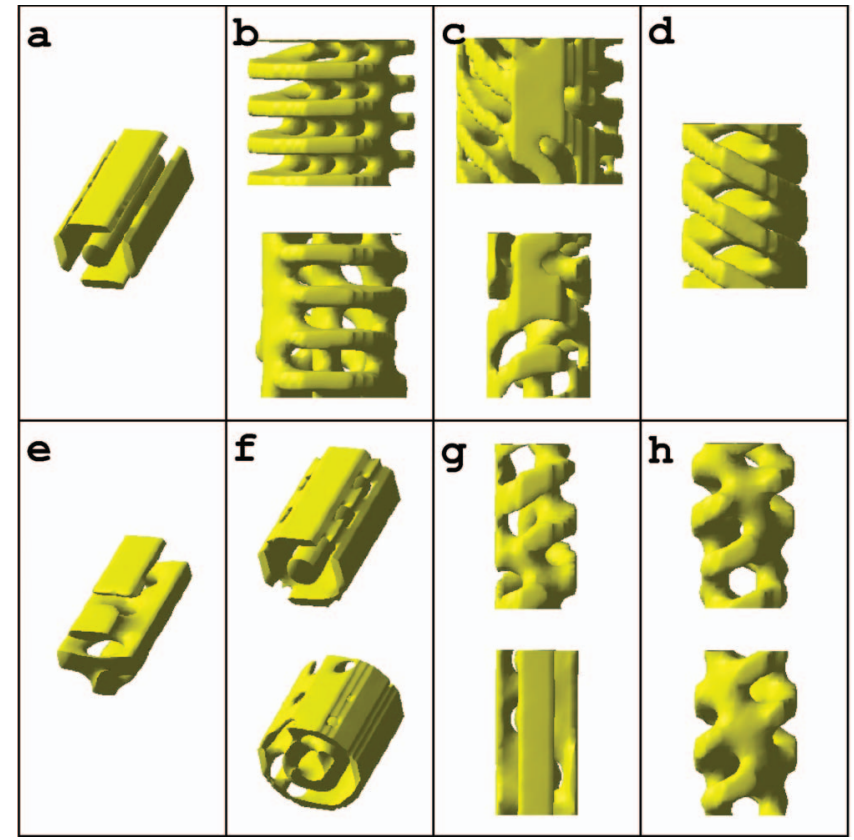

FIG. 4. (Color) Representative 3D nanopore structures for (a) $d L_{\|}$, (b) $d L_{\perp}$, (c) $d L_{\perp}^{\text {tilt }}$, (d) $L_{\perp}^{\text {tilt }} / L_{\perp}^{\text {tilt }}$, (e) $d L_{\perp, \|}$, (f) $d P L$, (g) $d D H$, and (h) $d G H$. The corresponding locations in the structure diagram are (a) $(0.93,-0.5)$, (b) $(2.21$, $0.3)$ and $(1.74,0.4),(\mathrm{c})(2.21,-0.1)$ and $(1.05,-0.1)$, (d) $(1.28,0.1)$, (e) $(0.93,0.0)$, (f) $(1.05,-0.3)$ and $(1.74,-0.2),(\mathrm{g})(0.81,-0.1)$ and $(0.81$, $-0.3)$, and (h) $(1.05,0.3)$ and $(1.05,0.4)$. Isodensity surfaces of the $S$ component for mean value are shown. 


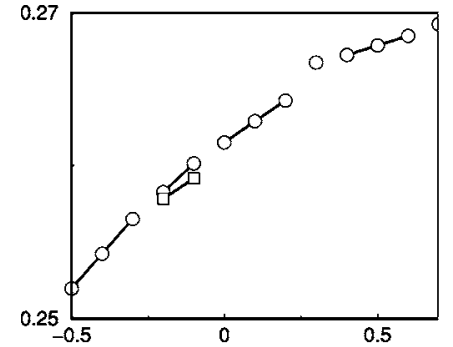

(a)

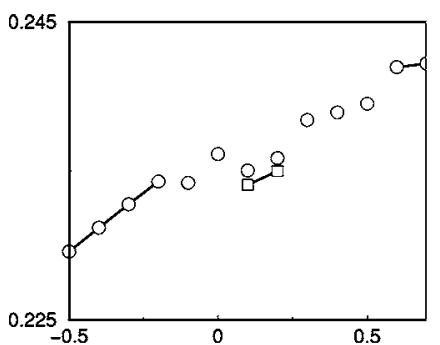

(d)

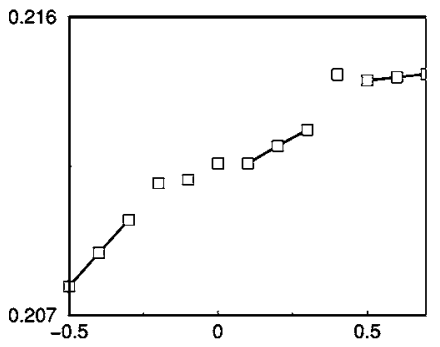

(g)

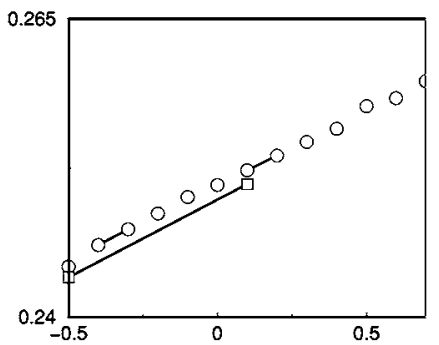

(b)

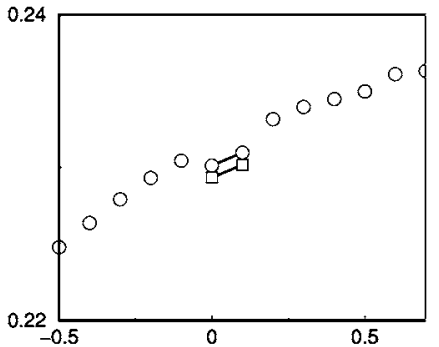

(e)

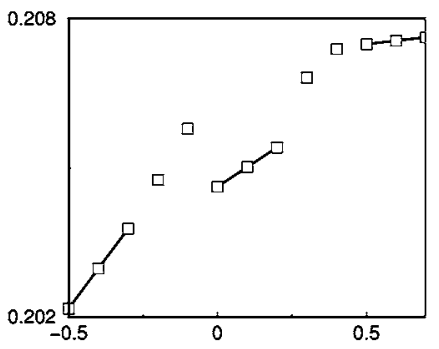

(h)

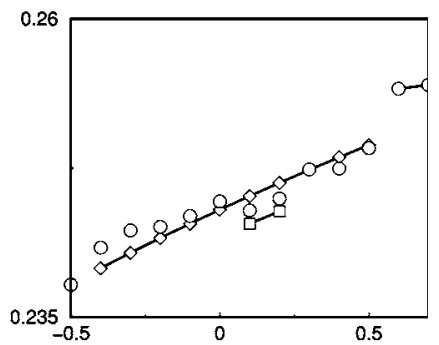

(c)

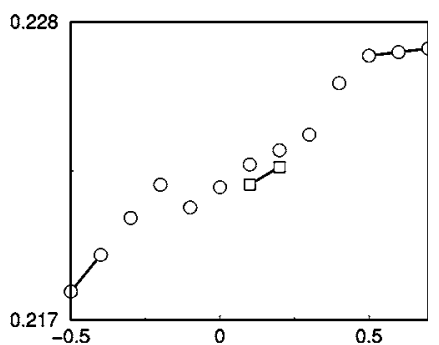

(f)

FIG. 5. Free energy (vertical axis) vs surface field strength $\xi$ (horizontal axis) for the reduced radii considered: (a) 0.58, (b) 0.70, (c) 0.81, (d) 0.93, (e) 1.05, (f) $1.28,(\mathrm{~g}) 1.74$, and (h) 2.21 . The free energies associated with the structures in Fig. 2 are denoted by open circles (for $L_{x}=32$ ) and open squares (for $L_{x}$ $=34$ or 36). Similar structures are connected by straight lines to guide the eyes. The open squares in (a)-(f) are associated with extra calculations (in all cases $L_{\perp}$ ) for different $L_{x}$ : (a) 34, [(b)-(e)] 36, and (f) 34. In (c) the free energy associated with the $D H$ structure $(\xi=-0.5)$ is calculated for other $\xi$ (open diamonds).

defected structures are remarkably stable. Based on visual inspection, and knowledge about metastable intermediate states along the pathway of formation of the perfect structures, we assign a symmetry group preceded by the letter $d$ (for instance, $d D H$ is a defected double helix). Other new structures will be discussed and annotated as they appear.

Focusing on the kinetic pathway, we remark that all structures in the diagram of Fig. 2 were obtained following a diffusive pathway [Eq. (5)]. Earlier work showed that microphase separation in confined systems often starts close to the pore wall. ${ }^{37}$ For the pore dimensions considered here, this effect will be small, but, especially for stronger surface fields, surface reconstructions will initially form, resulting in overall coverage of the pore wall by a single component. The transition to more stable structures with a different surface coverage (for instance, helices or stacked circles) requires considerable transport of material away from the wall, into the center of the nanopore. The fundamental mechanism of this transition is important, as it may hint when and where this process may be kinetically trapped. First, we consider which of the calculated structures is an equilibrium structure. For varying $\xi$, the free energy (see Fig. 5) associated with a particular structure (defined by $\rho_{S}$; due to incompressibility, $\rho_{B}$ is then also fixed) is given by [see also Eq. (1)] $F^{\text {tot }}=F$ $+F^{\text {wall }}=F\left[\rho_{S}\right]+\xi \mathcal{F}\left[\rho_{S}, \rho_{M}\right]$. Since both $F\left[\rho_{S}\right]=a$ and
$\mathcal{F}\left[\rho_{S}, \rho_{M}\right]=b$ have a constant value for a particular $\rho_{S}\left(\rho_{M}\right.$ is the mask field, and fixed by definition) the free energy values for this structure and varying surface field can be found on a line defined by $a+\xi b$. We only need two data points (or the values of $a$ and $b$ ) for the same structure to determine this line. However, we have to be careful using this procedure as structures with the same morphology type may differ in detail (and therefore in the values of $a$ and $b$ ), such as geometrical quantities and the degree of segregation. For instances, interpolation suggests that the $L_{\perp}$ structures for $\xi=-0.4,-0.3$ and $\xi=0.1,0.2$ (both for $R=0.70 L_{0}$ ) are different, although there is no structural difference in terms of easy computable geometrical quantities. Only when we consider the bare density values we find that the maximum of the concentration field $\theta_{S}(\mathbf{r})$ for $\xi=-0.4,-0.3$ is slightly higher than for the equivalent structures at $\xi=0.1,0.2$.

\section{Structure diagram and stability}

The structure diagram is shown in Fig. 2, and is indeed not symmetric. Equilibrium structures are distinguished by a gray background; this determination is based on the interpolation approach described above. Considering the general features of this diagram, one observes that several structures in this range are metastable. For large $R / L_{0}$, only $L_{\|}$and $L_{\perp}$ 
TABLE I. Radial distance between $S$ - $B$ or block-surface interfaces (denoted by $D_{i}, \Sigma_{i} D_{i}=R$, counted from the pore surface) in parallel structures for $\xi=-1.0$ and $\xi=0.7$. The position $\bar{R}$ of the interfaces is determined by the condition $\theta_{S}(\bar{R})=\theta_{B}(\bar{R})$.

\begin{tabular}{ccccccc}
\hline \hline$R / L_{0}$ & Structure & $D_{1}$ & $D_{2}$ & $D_{3}$ & $D_{4}$ & $D_{5}$ \\
\hline 0.58 & $P L(S B)$ & 0.5 & 4.5 & & & \\
0.70 & $P L(S B)$ & 1.6 & 4.4 & & & \\
1.74 & $L_{\|}(S B S B)$ & 1.5 & 4.9 & 4.1 & 4.5 & \\
0.81 & $L_{\|}(S B S)$ & 1.2 & 3.4 & 2.4 & & \\
0.93 & $d_{\|}(S B S)$ & 1.4 & 3.6 & 3.0 & & \\
1.05 & $L_{\|}(S B S)$ & 1.3 & 4.2 & 3.5 & & \\
1.28 & $L_{\|}(S B S)$ & 1.5 & 5.0 & 4.5 & & \\
2.21 & $L_{\|}(S B S B S)$ & 1.5 & 4.8 & 4.1 & 4.8 & \\
0.58 & $L_{\|}(B S)$ & 1.3 & 3.7 & & & \\
0.70 & $P L(B S)$ & 1.8 & 4.2 & & & \\
1.74 & $L_{\|}(B S B S)$ & 1.8 & 4.2 & 4.9 & 4.1 & \\
0.81 & $L_{\|}(B S B)$ & 1.4 & 3.1 & 2.5 & & \\
0.93 & $L_{\|}(B S B)$ & 1.4 & 3.3 & 3.3 & & \\
1.05 & $L_{\|}(B S B)$ & 1.5 & 3.7 & 3.8 & & \\
1.28 & $L_{\|}(B S B)$ & 1.8 & 4.4 & 4.8 & & \\
2.21 & $L_{\|}(B S B S B)$ & 1.8 & 4.0 & 4.9 & 4.0 & \\
\hline \hline
\end{tabular}

are stable; for small $R / L_{0}$ also other structures can be stable. The effect of incommensurability is limited, since we do not observe outliers of perpendicular structures for the larger radii considered (where the effect of the surface field is relatively small). However, for positive $\xi$ (pore surface likes $B$ ) and $R / L_{0}=1.28$ or 2.21 , the $L_{\|}$structure is only metastable and the transition to equilibrium structures trapped. Overall, this suggests that the pore radii $R / L_{0}=1.28$ and 2.21 are incommensurate, while $R / L_{0}=1.74$ is commensurate. Moreover, we see that for negative $\xi$ (pore surface likes $S$ ), $L_{\|}$is stable for $\xi \leqslant-0.4$, independent of the radius $R / L_{0}$. This asymmetry is due to the curvature effects and asymmetric packing frustration mentioned above.

Focusing on specific structures, we see that for large absolute values of $\xi$ surface reconstructions, $P L, d P L, d L_{\|}$, $L_{\perp, \|}$, and $L_{\|}$, dominate. In particular, we find $P L$ and $L_{\|, 1}(S B$ or $B S)$ for $R / L_{0} \in\{0.58,0.70\}, L_{\|, 1(1 / 2)}(S B S$ or $B S B)$ for $R / L_{0} \in\{0.81,0.93,1.05,1.28\}, L_{\|, 2}(S B S B$ or $B S B S)$ for $R / L_{0}=1.74$, and $L_{\|, 2(1 / 2)}(S B S B S$ or $B S B S B)$ for $R / L_{0}=2.21$. An analysis of domain spacing is given in Table I; the values are derived from the interface locations. Disconnected concentric cylinders $d L_{\|}$, where the cylinder close to the pore surface is broken up into four disconnected parallel lamellar patches, only appear for $R / L_{0}=0.93$. The distance between the patches increases with increasing $\xi$ in this region. The $L_{\perp, \|}$ is the same as the structure in Fig. 8b in Feng Ref. 23, and related to $d L_{\|}$(see discussion later). The number of perforations in $P L$ can vary and show hexagonal ordering when considered in the 2D plane. Geometrically, the $P L$ structure can be seen as an intermediate between $L_{\|}$and $H$. Defected perforated lamellae $d P L$, distinguished from $P L$ since the perforations do not show ordering on a larger scale, are found on the boundary between parallel and perpendicular structures. In response to the perforations the central cylinder sometimes adapts an oval cross section. Defected $P L$ only appears for negative $\xi$ (surface preference to $S$ ). The majority of surface reconstructions $\left(P L\right.$ or $\left.L_{\|}\right)$for positive $\xi$ (surface preference to $B$ ) are not stable structures. They are examples of kinetically trapped structures, due to the presence of the pore surface, which gives rise to $L_{\|}$related structures in the early stages of phase separation.

Perpendicular structures, tilted tacked disks $L_{\perp}^{\text {tilt }}$ and $L_{\perp}$ (four disks for all $L_{x}$ considered), are found in the center of the diagram, for small $\xi$. For small relative radii $R / L_{0}$, the disks are perpendicular to the pore surface, $L_{\perp}$. For larger $R / L_{0}$ there are only three disks, and they are tilted with respect to this surface, $L_{\perp}^{\text {tilt }}$. For the largest pore radii, $R / L_{0}$ $=1.74$ and 2.21, the pore length $L_{x}\left(L_{x}=34=3.95 L_{0}\right.$ and $L_{x}$ $=36=4.19 L_{0}$, respectively) is somewhat larger than the standard $L_{x}=32$, and the structure is $L_{\perp}$. The $L_{\perp}^{\text {tilt }} / L_{\perp}^{\text {tilt }}$ structure are two coexisting perpendicular $L_{\perp}^{\text {tilt }}$ structures, with opposite tilt angles. The details of these mixed structures, not representing equilibrium structures for obvious reasons, will be considered later.

Helical structures, $H, D H$, and a new $G H$ structure, are found at the rather broad boundary between perpendicular and parallel structures. These structures possess features relating them to both perpendicular and parallel structures: The axis of winding is parallel to the pore axis, and the wall coverage is nonuniform. The $G H$ structure in $(1.05,0.4)$ is related to the helical structures, but differs topologically since small helical patches are threefold connected with helical patches on the opposite side, and these connections join into two cylinders parallel to the pore axis. The $G H$ for $(1.16,0.5)$ and $(1.40,0.4)$ share this property, but instead the connections form two lamellar patches and three cylinders, respectively. We call this structure gyroid-helical $(G H)$ because of the threefold connectivity. Stable helices (left and right hand) and a double helix are formed for $R / L_{0}<1$ and negative $\xi$. The helical structures for positive $\xi$ are all metastable. Metastable $G H$ are only found for larger effective radii, $R / L_{0}>1$, adjacent to both parallel and perpendicular structures.

Several coexisting structures, $L_{\perp} / P L, L_{\perp}^{\text {tilt }} / H, L_{\perp}^{\text {tilt }} / G H$, 
a)
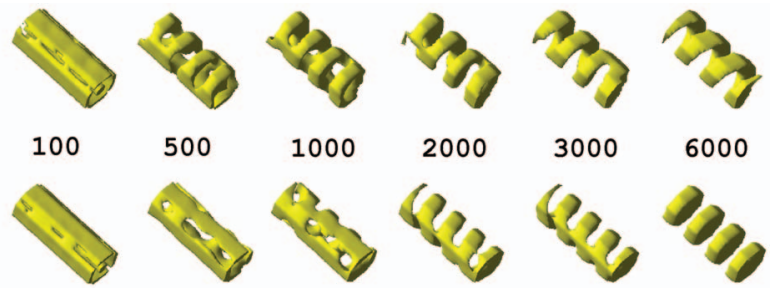

b)
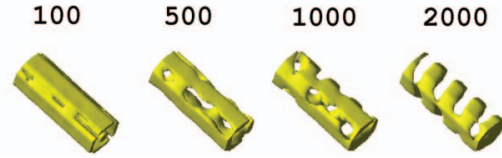

FIG. 6. (Color) The effect of extensional stress. Kinetics of nanopore structure formation for $R / L_{0}=0.70, \xi=-0.5$, and varying pore length $L_{x}$. (a) $L_{x}$ $=32$ (stable structure: $H$ ) and (b) $L_{x}=36$ (stable structure: $L_{\perp}$ ). The images show isosurfaces for the $S$-block, for isosurface value $\bar{\theta}_{S}$. The numbers indicate the dimensionless time steps TMS.

$L_{\perp}^{\mathrm{tilt}} / H$, and $L_{\perp} / L_{\|} ;$and defected structures, $d L_{\perp}^{\mathrm{tilt}}, d L_{\perp}, d G H$, and $d L_{\perp, \|}$ are found directly adjacent to their "perfect" counterparts, and stay defected after many simulation time steps (TMS). Apparently, the driving force for the removal of different types of defects in these structures is rather small, and as a result the structures are kinetically trapped. The only exceptions, coexisting $H / P L$ and $P L / L_{\|}$for positive $\xi$ that are not adjacent to a $P L$ structure, show that $P L$ is associated with a free energy close to the one for $H$ and $L_{\|}$, respectively. For $R / L_{0}=0.81$ and $-0.4 \leqslant \xi \leqslant 0.0$ the structures are very defected, and remain as such, even after a large number of extra timesteps (TMS).

\section{The value of $L_{x} / L_{0}$ : Elongational stress and perpendicular structures}

Earlier work for a "soft" confined system ${ }^{35}$ concluded that the $L_{\perp}, L_{\perp}^{\text {tilt }}$, and $H$ structures are related. Their stability depends on the extensional force on the system, originating either from an external field (experiments) or from boundary conditions (computations). Extensional forces may be present in experimental and computational studies dealing with structure formation in nanopores, but have not received much attention so far. The only exception is a computational study in the strong segregation limit (SSL), ${ }^{25}$ where MC based on a grand canonical ensemble was employed. Especially in computational methods considering a canonical ensemble and a single $L_{x}$, this type of commensurability issues cannot always be avoided. We argue that a deeper understanding of this effect is relevant. In experiments, for instance, extensional forces play a role when the pore surface is very rough, the pore length is very small (often the case in applications considered in soft nanotechnology), and when shear fields are present, as is the case in a recently developed experimental technique for the fabrication of nanowires. ${ }^{38}$ In the present study, we have considered this effect by varying $L_{x}$ for a small set of selected parameters: $L_{x}=34=3.95 L_{0}$ for $(0.58,-0.1)$ and $(1.28,0.1)$, and $L_{x}=36=4.19 L_{0}$ for $(0.70$, $-0.5),(0.81,0.2),(0.93,0.2)$, and $(1.05,0.1)$. In all cases the microstructure evolves into $L_{\perp}$ (four disks), with an associated free energy that is lower than for the original structure for $L_{x}=32$. A detailed analysis of the structure evolution revealed that $L_{\perp}^{\text {tilt }}$ and $H$ structures can also be kinetically related to $L_{\perp}$ : In some cases, they mediate large-scale structural reorganization. This phenomenon can be observed from the helicity that appears during the evolution of the $L_{\perp}$ struc-

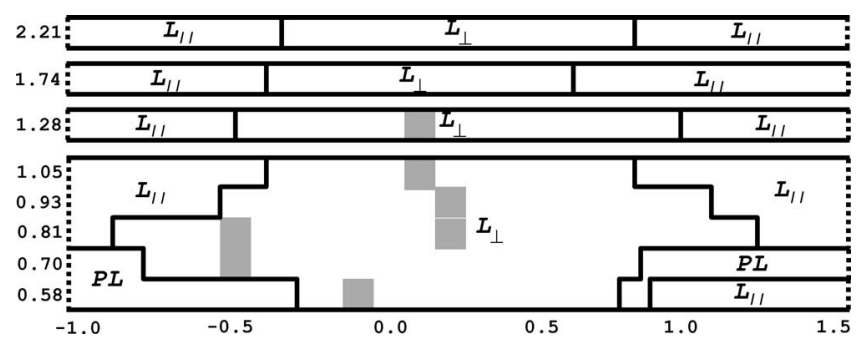

FIG. 7. Nanopore phase diagram for varying pore radius (in $R / L_{0}$ ) and surface field (in $\mathrm{kJ} / \mathrm{mol}$ ). Stable structures for each $R / L_{0}$ were determined using all free energy data in Fig. 5, irrespective of the pore lengths $L_{x}$. The absence of $L_{\|}$for $\xi<0(R=0.58,0.70)$ and $\xi>0(R=0.70)$ does not allow for the analysis of the boundaries of the $P L$ region.

ture in a pore of $L_{x}=36$ and $\left(R / L_{0}, \xi\right)=(0.70,-0.5)$ (see Fig. 6 for the details of the formation pathway for different $L_{x}$ ). It is in agreement with earlier findings for a fully symmetric $A B$ diblock in Ref. 13.

Apart from the single additional calculation for each of pores and $L_{x} \neq 32$, we used the $L_{\perp}$ structures to compute the free energy for an additional surface field strength $\xi$. Interpolation between these two values enables us to reconsider the stability of the structures in Fig. 2 with respect to $L_{\perp}$. We make two general remarks: (i) Although the interpolation technique is valuable for comparing the stability of different microstructures in pores of equal length (for varying $\xi$ ), in principle this procedure cannot be used to differentiate between microstructures in pores of different lengths. For instance, an instantaneous change of the pore length (or equal, extensional force) during the evolution gives rise to a deformation of the structure, and possibly a transition to a more stable structure. However, in contrast to SCF techniques, DDFT does not impose symmetry, and changes in free energy associated with a deformation of an existing structure, for instance, an affine deformation of a helical structure, cannot be quantized directly. Instead, additional calculations are necessary for many alternative structure types and are very time consuming in general. ${ }^{35}$ We therefore anticipate on the results of the SSL study, ${ }^{25}$ which considered nanopore structure formation in the absence of elongational stress. This study identified, besides lamellar structures, only stable $P L$. Consequently, we adapt a practical approach and use all data obtained by simulation (see Fig. 5), independent of $L_{x}$, to suggest a phase diagram of equilibrium structures (see Fig. 7), without considering the deformability of the nonlamellar structures. We note that the stability of the $P L$ phase could not be determined indefinitely, since the alternative $L_{\|}$phase was not formed for these small radii. Only for $R / L_{0}=0.58$ and positive $\xi$, concentric cylinders $(B S)$ were formed, and the region where $P L$ is equilibrium structure could be determined. (ii) The additional simulations for $L_{x} \neq 32$ were carried out for relatively weak surface fields, where perpendicular structures are likely to form. Interpolation indicates (see Fig. 7) that the equilibrium structure can be perpendicular even for relatively strong surface fields, for instance, due to incommensurability along the radial direction. However, the large structural rearrangements required for the transition to (stable) perpendicular structures, starting from the (unstable) parallel structures that are initially formed due to the strong 


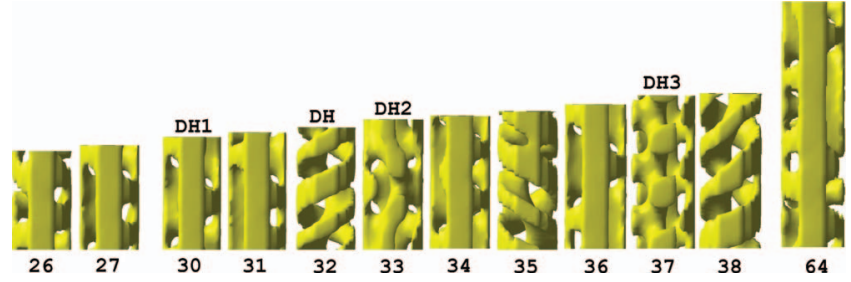

FIG. 8. (Color) The effect of extensional stress for the double helical structure $\left(R / L_{0}=0.81, \xi=-0.5\right)$. Upon a variation of the pore length $L_{x}$ a number of metastable structure classes [denoted by $\mathrm{DHi}(i=1-3)$ ] were identified. The images show isosurfaces for the $S$-block, for isosurface value $\bar{\theta}_{S}$. Numbers indicate the pore length $L_{x}$ (in grid units).

surface interaction, may lead to arrested structures, i.e., microstructures that are kinetically trapped in metastable states. A good example is the $D H$ structure in $(0.81,-0.5)$. Although the phase diagram of Fig. 7 shows that $\ell_{\perp}$ is the equilibrium structure, we challenged the stability of the $D H$ structure by choosing a number of systems with different pore lengths $L_{x}$ (each system was quenched from a homogeneous mixture at TMS $=0$, see Figs. 8 and 9). We never obtained the equilibrium structure as a result of the dynamic pathway. Instead, upon variation of the extensional pressure, we obtain structures with an overall parallel orientation that lack symmetry on a larger scale. Only for $L_{x}=32,35$, and 38 we find an almost perfect $D H$ structure. We rank these other structures in three different classes, labeled as $\mathrm{DHi}$. Structure DH1 can be seen as a highly interconnected and defected $L_{\perp, \|}$ structure, where the center lamella contains large holes and is connected to the other structure. Structure $\mathrm{DH} 2$ is completely symmetric; the axis of symmetry is $L_{x} / 2$. Structure $\mathrm{DH} 3$ consists of two disconnected sheets of different structures, one similar to $P L(B)$ and the other similar to $P L(S)$. For $L_{x}=64$ the structure is very defected and a combination of other structures. Comparing the free energies in Fig. 9, we find that the one of the metastable $D H$ structure $\left(L_{x}=32\right)$ is the lowest, indicating that the extensional force is minimal for $L_{x}=32$ and the structure is trapped due to the diffusive kinetic pathway. The finding of $D H$ for other $L_{x}$ shows that the factor $3 / L$ (with $L$ the particular spacing of

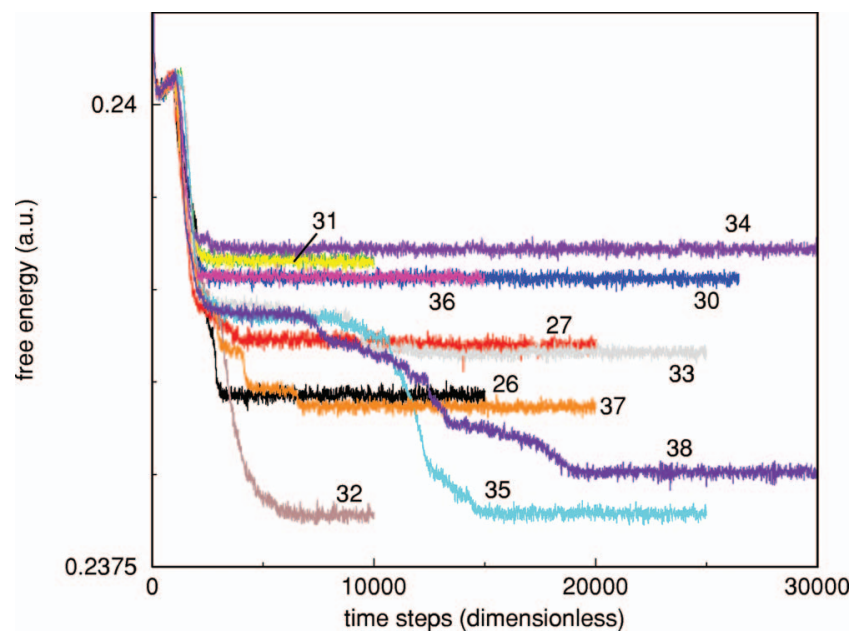

FIG. 9. (Color) Evolution of the free energy associated with the structures in Fig. 8. Numbers indicate the pore length $L_{x}$. the structure) plays an important role. In general, we see that an increase of the nanopore length slows down the separation dynamics. Close examination of the structures in diagram 2 reveals that the calculated structures for $-0.4 \leqslant \xi \leqslant 0.0$ are defected $D H$ or resemble the intermediate $D H 1$. In all cases, the kinetics disables the mass transport necessary for the $D H \rightarrow L_{\perp}$ transition.

\section{The value of $R / L_{0}$ : Commensurability and parallel structures}

From the phase diagram in Fig. 7 (see discussion for the derivation of this diagram above) we distinguish two specific values: $R=0.81 L_{0}$ and $R=1.74 L_{0}$. For $R=0.81 L_{0}$, the pore radius $R<L_{0}$ and the surface field is therefore strong, but confinement effects apparently prevent the formation of parallel structures, in favor of the $L_{\perp}$ structure, up to large surface field strengths. Since this radius also marks the transition of $S B\left(0.70 L_{0}\right)$ to $S B S$ (or $B S$ to $B S B$ ), we conclude that $R=0.81 L_{0}$ is incommensurate. For $R=1.74 L_{0}>L_{0}$, the surface field influence is much less. Apart from a symmetric $L_{\|}$ region for large surface fields (we note that the number of $S$ and $B$ domains is the same, independent of the wetting block), only a small region of perpendicular structures is observed for almost neutral pores, and we conclude that the pore size is commensurate. We consider the simulation results in Fig. 2 in more detail. Domain distances are shown in Table I and are inexact for the $P L$ structure, which is not radial symmetric. For the smallest $R=0.58 L_{0}$, parallel structures are found for strong surface fields. Most of these structures are $P L[P L(S)$ or $P L(B)]$, and $L_{\|}$is only found for $(0.58,0.7)$. The interpolation procedure showed that, at the onset of the $L_{\|}$region, the free energies associated with both parallel phases are relatively close (the value of $b$ is almost equal, see discussion of the interpolation procedure before), which explains that $L_{\|}$is found for $\xi=0.7$ due to kinetic trapping. Upon comparing the $P L$ and $L_{\|}$structures geometrically, we see that the formation of necks on the central cylinder in $P L$ leads to an increased curvature of the $S$ - $B$ interface and contact area of the nonpreferred block with the pore surface. If we compare the position of this $S$ - $B$ interface for this incommensurate and the commensurate situation $\left(R / L_{0}\right.$ $=1.74, S$ - $B$ interface closest to the pore center, see Table I) we observe a reduced thickness for positive $\xi$ and equal thickness for negative $\xi$. This illustrates the curvature effect mentioned before, meaning that the elastic chain deformation associated with the formation of $L_{\|}$can much easier be facilitated when the interface is curved towards the shortest $S$-part of the chain, than to longest $B$-part. The same phenomenon can be observed for $R=0.70 L_{0}$. Here, concentric cylinders $\left(L_{\|}\right)$are completely absent in the simulated range. Alternative $P L$ structures, very similar to the ones for $R$ $=0.58 L_{0}$ apart from the number of perforations, can be found, but equilibrium $P L(S)$ structures are only found for rather low $\xi$ values, again due to the curvature effect. In order to fill the pore, the domains are rather extended (Table I). The formation of parallel structures requires strong stretching of the part of the chain that contains the wetting blocks, giving rise to an entropic penalty that is only counterbalanced for stronger surface repulsion. For $R=0.81 L_{0}$ 
a)

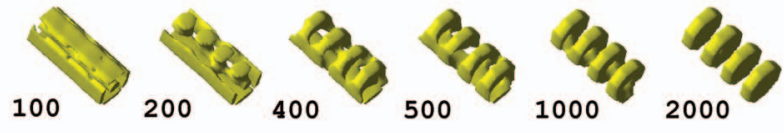

b)

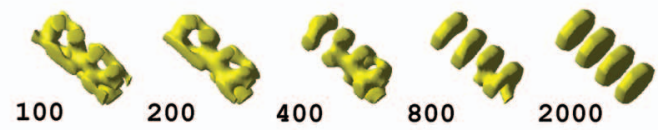

c)

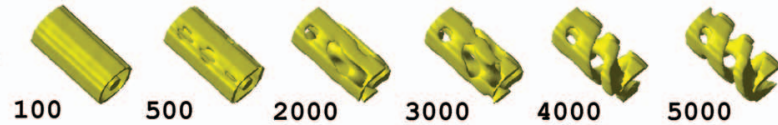

d)

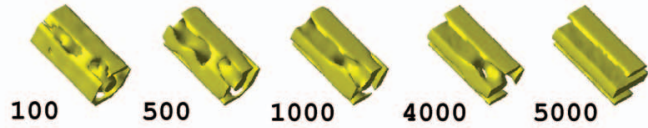

e)
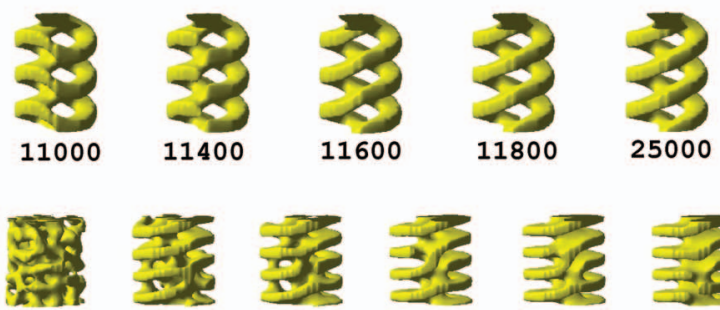

f)

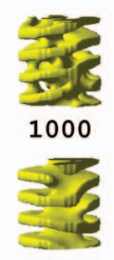

8000

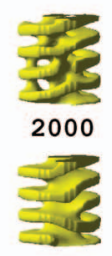

10000

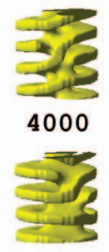

15000

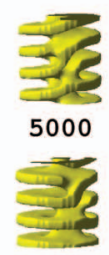

20000

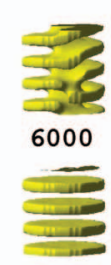

25000

FIG. 10. (Color) Kinetics of nanopore structure formation for selected pore radii and surface field strength (in $\mathrm{kJ} / \mathrm{mol}$ ): (a) $R / L_{0}=0.70, \xi=-0.4$, (b) $R / L_{0}=0.70, \xi=0.1$, (c) $R / L_{0}=0.81, \xi=-0.5$, (d) $R / L_{0}=0.93, \xi=-0.1$, (e) $R / L_{0}=1.28, \xi=0.2$, and (f) $R / L_{0}=1.74, \xi=0.3$. The images show isosurfaces for the $S$-block, for isosurface value $\bar{\theta}_{S}$. The numbers indicate the dimensionless time steps TMS.

surface reconstructions $L_{\|, 1(1 / 2)}(S B S$ or $B S B)$ are found and $P L$ is absent, apart from the coexisting $H / P L$ structure $(\xi$ $=0.5$ ). However, from the phase diagram, we conclude that all surface reconstructions $L_{\|}$are metastable and formed due to kinetic factors. The analysis of the domain distances in Table I shows rather small domain sizes, and therefore strong compression of the chains. Although $L_{\|, 1(1 / 2)}$ is only found for positive $\xi\left(R=0.93 L_{0}\right)$, we observe many other parallel structures, suggesting that this radius $R$ is somewhat commensurate. However, from the analysis in Table I we see that the domain spacing for this radius is still rather small (compared to bulk spacing, and the spacing of $D_{3}+D_{4}=9.0$ found for the inner two layers in the commensurate $B S B S$ structure for $\xi=0.7$ and $\left.R / L_{0}=1.74\right)$, especially towards the center. Moreover, the equilibrium structure in this range is $L_{\perp}$ (see Fig. 7). We focus on the disconnected lamellar patches $\left(d L_{\|}\right)$ for $\xi \leqslant-0.2$. Because of the masking procedure, the cross section of the pore is not completely circular, and the curvature of the pore wall varies. Hence, one could expect that metastable concentric cylinders formed due to kinetic factors, and that the origin of these disconnections, appearing in regions of high curvature, is the chain-stretching required for the formation of undefected $L_{\|}$. Close examination of the

kinetic pathway towards the $L_{\perp, \|}$ structure [Fig. 10(d)] shows $d L_{\|}$as an intermediate structure. In particular, along the pathway two of the lamellar patches and the central cylinder have merged to form the center lamellae. In the early stage, undulations lead to connections between the different structural elements in $d L_{\|}$(very fast). Subsequently, these connections merge (and others are removed) by a complex process that includes the formation of holes (very slow). The structures along this pathway resemble the metastable $D H 1$ structures that are found in $(0.81,-0.5)$ for varying $L_{x}$. Finally the $L_{\perp, \|}$ structure forms. Apparently the removal of defects depends on the surface field strength and is slowed down considerably by an increase of $\xi$ from -0.1 to 0.0 . In MC calculations $^{23}$ the $L_{\perp, \|}$ structure was also found for a pore radius $R \approx L_{0}$. It was suggested that it represents an intermediate between the $L_{\|}$and $L_{\perp}$ structures, but no further explanation was given. The stability analysis suggests that all nonperpendicular structures are metastable. We claim that this structure represents a kinetically arrested alternative for stable $L_{\perp}$. Both structures are formed for small to vanishing surface fields, in order to avoid the elastic chain deformation due to the curved $S-B$ interfaces in concentric cylinders. Following the same reasoning, the $d L_{\|}$structures (instead of $L_{\|}$) for $\xi<-0.1$ are metastable structures along the pathway to this $L_{\perp, \| l}$ structure, and kinetically trapped since the undulations necessary for the formation of this structure are suppressed by the strong surface field.

For $R=1.05 L_{0}$ the situation is weakly incommensurate, and $L_{\perp}$ is the equilibrium structure between $-0.4 \leqslant \xi \leqslant 0.8$. This can also be seen from the slightly perturbed spacing in Table I for $\xi=-1$. For $-0.5 \leqslant \xi \leqslant-0.3$ in Fig. $2(d P L)$ holes are formed in the region of highest curvature, and we find that the density field in the center of the pore is slightly higher for $\xi=-0.5$ than for $\xi=-1$ (no holes). This $d P L$ structure was also observed as the initial stage in the formation of $D H, H$, and $L_{\perp}$ structures for these $\xi$ and smaller $R$, and for the slow transition to $d L_{\perp}^{\text {tilt }}$ for $\xi=-0.2,-0.1$, where we simulated up to 40000 TMS. For positive $\xi$, the parallel structures are also metastable, but the free energy associated with the $P L(B) / L_{\|}$coexisting structure $(\xi=0.6)$ is slightly below the one extrapolated for perfect $L_{\|}$. This is once more an indication that the chain deformation associated with concentric cylinders can be relieved by the formation of necks, and therefore $P L$ is more stable. Moreover, this transition from $L_{\|}$ to $P L$ (and eventually the transition, via other intermediate structures, to $L_{\perp}$ ) is obviously very slow. The parallel structure $L_{\perp, \|}$ is absent, showing that the kinetic pathway leading to this metastable structure is rather unique.

For $R=1.28 L_{0}$, the phase diagram suggests that the pore size is slightly too large for $L_{\|, 1(1 / 2)}$. The detailed analysis in Table I shows that the chains in the center of the pore are stretched, compared to the bulk domain thicknesses $\left(D_{B}\right.$ $=4.7$ and $\left.D_{S}=3.9\right)$, and to the $L_{\|, 1(1 / 2)}$ structures for other $R$. The boundary for the equilibrium $L_{\perp}$ phase shifts to stronger surface fields, but this shift is small compared to $R=1.05 L_{0}$. The larger shift for positive $\xi$ is due to the curvature of the $S$ - $B$ interface of the central cylinder, which is curved towards the shortest block for negative $\xi$.

For $R=1.74 L_{0}$ surface reconstructions $L_{\|, 2}$ are found for 
positive and negative $\xi$. Moreover, only at the boundary with defected structures, these surface reconstructions are metastable. The stability region of $L_{\|}$is almost symmetric around $\xi=0.1$, and we conclude that the situation is commensurate. The chains are slightly stretched (see Table I) compared to the spacing to the domain spacing in the bulk due to curvature effects. A comparison of the spacings in pores for $R / L_{0}=1.74$ and 2.21 suggests that especially the chains in the center of the pore are compressed for the largest pore. The driving force for the transition of the mestastable surface reconstructions to $L_{\perp}$ is rather small (see Fig. 5).

\section{Detailed kinetic pathways}

Although some kinetic pathways have been considered in the previous subsections, we focus on a few more representative pathways here. For $R=0.70 L_{0}$, different kinetic pathways were found leading to the final $L_{\perp}$ structures for $\xi=-0.4$ [Fig. 10(a)] and $\xi=0.1$ [Fig. 10(b)]. For the negative $\xi=-0.4$ the initial structure is $L_{\|}$. At later stages (200 TMS) the cylinder in the center of the pore has broken up to form short and disconnected cylinders, while part of the outer curved lamellae stays intact. Remarkably, parts of this outer curved lamellae remain intact during the transition and mediate the mass transfer from the wall into the pores interior. This is the source of the toroidal structures that appear as intermediate, at the positions where the stacked disks are later formed. For the positive $\xi=0.1$ the initial structure is bicontinuous [or very defected $P L(S)$ ] with many threefold connections in the center of the pore. These connections can be seen as defects, as very early in the evolution there is an excess of material at positions where stacked disks are later formed. Fourfold connections (small lamellar patches) and helical domains mediate the mass transfer needed for the formation of perfect stacked disks in this case.

The most interesting structure for $R=0.81 L_{0}$ is the double helix $(D H)$ for $\xi=-0.5$. Figure $10(\mathrm{c})$ shows that the formation of this structure is a rather slow process, and starts from $L_{\|}(100 \mathrm{TMS})$. In the early stages, equidistant perforations form in the outer curved lamellae, similar to the ones formed for $R / L_{0}=1.05$ and $-0.4 \leqslant \xi \leqslant-0.3$. These perforations grow in time while the cylinder in the center of the pore undulates and breaks up to form a bicontinuous network. In contrast to before this bicontinuous network itself forms the nucleus of the final structure, which is the double helix structure. Due to its helical nature, defect removal cannot be mediated by the formation and breakage of helical connections and thus the process is rather slow.

The metastable parallel structure $G H$ is only formed for positive $\xi$, and either stabilized by an extensional force or kinetically trapped along the pathway of the $L_{\|} \rightarrow L_{\perp}$ transition. Its finding adjacent to perpendicular structures (deformed $L_{\perp}$ ) and $L_{\|}$for $R=1.28 L_{0}$, or perpendicular structures and $P L$, coexisting with $L_{\|}$and $H$, for smaller pores suggests that it may evolve from $P L$. Close examination of the pathway for $R / L_{0}=1.28(\xi=0.4)$ and $R / L_{0}=0.93(\xi=0.3)$ shows that the initial structure $L_{\|}$rapidly transforms (after 100 TMS) into a very defected structure, where the perforations in the outer $B$ layer are uneven and randomly positioned.
Since $P L$ only appears in coexistence with other structures for positive $\xi$, we conclude that the kinetic pathways for the formation of $G H$ and $P L$ must be different.

For $R=1.28 L_{0}$, we concentrate on the effect of elongational stress on the evolution of the $L_{\perp}^{\text {tilt }} / L_{\perp}^{\text {tilt }}$ structure, where the equilibrium structure is $L_{\perp}$. In particular, we consider the structure evolution for $\xi=0.2$ in detail, since incommensurability issues with respect to the pore radius are absent. First, we note that the cylinder in the center of the pore for $\xi=-0.2$ shows some undulations, but the number of pores in the outer layer remains constant for longer simulation times, and no connections with the inner structures are formed within the limits of simulation time. These connections are required for the transition/nucleation of one phase (in)to the other. For $\xi=-0.1$ a defected structure without apparent symmetry at TMS $=10000$ evolves into a helical phase at TMS $=25000$. Since the axis of winding is away from the center, we conclude that the structure can be seen as a majority $L_{\perp}^{\text {tilt }}$ under some angle (with the pore wall), connected to a minority $L_{\perp}^{\text {tilt }}$ under a reverse angle. As a natural consequence, the connections between these two partial structures have a helical nature. We observe similar structures for $\xi=0.1$ and $\xi=0.3$. For $\xi=0.2$ the axis of winding coincides with the center of the pore, and we could classify this structure as analogous to the $D H$ structure for smaller $R$. However, in line with the other structures for this radius this could be seen as a merger of two equally sized clusters of oppositely tilted and connected stacked disk morphologies. Moreover, in the evolution for $\xi=0.2$ [see Fig. 10(e)] we observe a fundamental mechanism: The orientation with respect to the pore wall of the total structure, as well as the change of chirality of local structure, are mediated by melting and reconnection of helical sections. In this way, unfavorable orientation and winding in part of the structure (for instance, giving rise to holes or increased curvature) can be avoided. We conclude that all these structures $-0.1<\xi<0.3$ are actually coexisting $L_{\perp}^{\text {tilt }}$ under two different angles. The helical connections between these two cluster structures are relatively stable in time, and analogous of the double periodic array of saddle surfaces found in lamella-forming thin films under an electric field. ${ }^{39}$ Apparently, they do not cost much energy to the system. This is confirmed by the free energy plot in Fig. 5, where the free energies associated with these different helical structures fall almost on a single line.

To see whether the elongational stress is the cause for this fundamental mechanism, we also considered the structural evolution in the absence of incommensurability along the pore, $\xi=0.3$ and $R=174 L_{0}$ [see Fig. 10(f)]. The detailed analysis confirms that the fundamental mechanism identified for formation of structure under elongational stress, i.e., reorientation on a higher structural level by helical connections, is also instrumental for the formation of equilibrium $L_{\perp}$ structures. One can clearly see that the tilted and interconnected disks that form in the initial stages (1000 TMS) slowly transform into perfectly stacked disks or $L_{\perp}(25000$ TMS). The mechanism is a moving front of helical connections, just like shown in Fig. 10(e). This effect can also be observed for $R=2.21 L_{0}$ and $\xi=0.0$ or 0.2 (not shown), where the initial coexisting structure $L_{\perp}^{\text {tilt }} / L_{\perp}^{\text {tilt }} \quad(\mathrm{TMS}=10000)$ 
evolves into $L_{\perp}$ (TMS $\left.=20000\right)$. From these observations we conclude that coexisting stacked tilted disks with helical domains are the preferred transient structure, independent of the elongational stress. The most probable cause is that interconnected tilted structures can much easier accommodate the necessary large-scale structural rearrangements that are necessary for the formation of $L_{\perp}$. Helical connections play an important role in the removal of defects, and slowly change the tilt of the intermediate structure, leading to the formation of equilibrium $L_{\perp}$ structures.

\section{Discussion: Comparison to other studies 1. Experiments}

Up to now, experimental groups have considered structure formation of (nearly) symmetric PS- $b$-PB (Refs. 16-19) and PS- $b$-PMMA (Ref. 20) confined in nanopores. Almost all observed experimental microstructures belong to the class of concentric cylinders. This does not come as a surprise, as for most diblock copolymers one of the blocks has a higher affinity for aluminum. We have previously concluded that, especially for the larger pore sizes considered, surface fields above a certain (relatively low) threshold value lead to socalled surface reconstructions: Concentric cylinders. In the experimental procedure, the block copolymers were introduced into the alumina nanopores at elevated temperatures via capillary action and subsequently cooled. After this procedure a weak base was used to dissolve the alumina and produced the freestanding rods of block copolymer that were imaged. The thus obtained rods show several features: The cross sections vary in shape (from ellipsoidal to spherical) and the thickness of the rod varies substantially along the rods (due to the roughness present in the alumina pores). As a result, the actual pore radius can only be determined approximately. Although some measurements of surface tension were performed, exact numbers quantifying the affinity of $P S, P B$, and $P M M A$ for the aluminium pore material are missing.

We conclude that the stacked-disk or toroidal-type structure in nearly symmetric PS- $b$-PB block copolymer ${ }^{16}$ is the best candidate for a true comparison between our calculations and experiments. We therefore choose the simulation parameters as close as possible to the values for this experimental system. The alternative structure was found at a temperature of $T=398.15 \mathrm{~K}$, above the glass transition temperature, ${ }^{17,19}$ in a system with a molecular weight of $M$ $=18400$ (the number of monomers $N \sim 250$ ). The experimentally measured repeat period $L_{0}=17.6 \mathrm{~nm}$, giving rise to an incommensurate pore radius $R / L_{0} \sim 1.3$. We note that the effective Flory-Huggins (FH) parameter $\chi$ depends to some extent on the details of the experimental system. Using earlier measurements by the same group we find $\chi=0.058$ (Ref. 40) and obtain $\chi N \approx 14.5$. For a coarse-grained chain with $N=22\left(A_{10} B_{12}\right)$ we therefore find an effective Flory-Huggins parameter $\chi_{S B}=0.66$. Here we consider $\chi_{S B}=0.6$ instead, and focus on the influence of reduced FH parameter in general (the calculations in the previous sections were carried out for $\left.\chi_{S B}=0.8\right)$. Although we use the bare FH parameters in the (a)
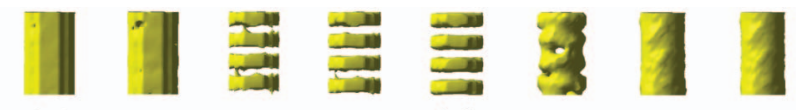

)

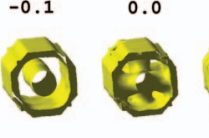

.

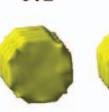

0.3

\section{4}

0.5

0.6
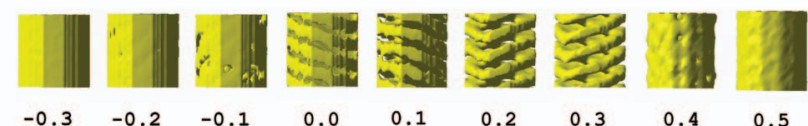

(b)
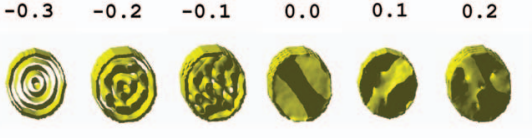

(4)

$0.4 \quad 0.5$

(c)
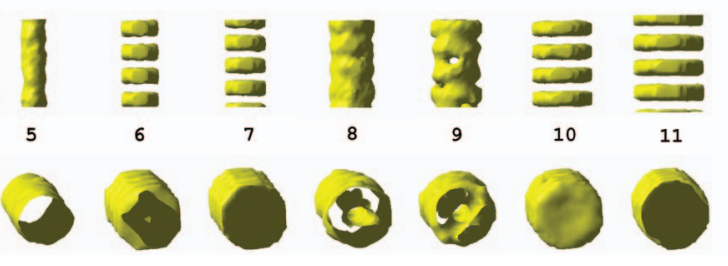

FIG. 11. (Color) Nanopore structures for $S_{10} B_{12}\left(\chi=0.6, L_{x}=32\right)$, matching the experimental system of Shin et al. (Ref. 16), (a) for pore radii $R=19$ ( $\left.L_{x}=36\right)$, (b) $R=9\left(L_{x}=32\right.$ ), and (c) surface field $-1.0 \leqslant \xi \leqslant 0.7$ (in $\mathrm{kJ} / \mathrm{mol}$ ). Parallel structures for surface fields higher than the threshold surface field strength are not shown. The structures in (c) are for constant surface field $(\xi=0.4)$ and pore radius varying between 5 and $11\left(L_{x}=32\right)$. For each radius, $R$, structures from two different viewing angles are shown. The images show isosurfaces for the $S$-block, for isosurface value $\bar{\theta}_{S}$.

calculations, the referring $\varepsilon_{S B}^{0}=1.5 \mathrm{~kJ} / \mathrm{mol}$ for the choice of the temperature $(T=300 \mathrm{~K})$ in the standard input in DDFT.

The results of calculations for $\chi_{S B}=0.6$ and two radii $R$ (and varying $\xi$ ) are shown in Figs. 11(a) and 11(b). We conclude that for the largest $R=19$ the effect of reduced $\chi$ is rather small. Comparing the results for $R=19$ in Fig. 11 and $R=2.21 L_{0}$ in Fig. 4 (for the same cylinder length $L_{x}$ ) we see that the phase boundaries have not been shifted much. For low and high values of $\xi$ concentric cylinders are found with an additional layer when compared to the results for higher block-block interaction. This finding is in well agreement with the 2D SCF calculations of Li et al. ${ }^{21}$ where a transition from $L_{3}$ to $L_{2}$ (notation of Ref. 21) is found for $\chi N>13$. The tilt of the $L_{\perp}$ for small $\xi$ reflects the different bulk lamellar distance $L_{0}$ associated with the change in $\chi_{S B}$ and is due to an extensional force. For the smallest $R=9$, concentric cylinders $\left(L_{\|}\right)$are found for $\xi \leqslant-0.1$ and $\xi \geqslant 0.5$, with the same number of layers as for larger $\chi_{S B}$. For $0.1 \leqslant \xi \leqslant 0.3$, a perfect $L_{\perp}$ structure is obtained. Constructing the lines associated with different structures shows that $\xi=0.0$ and $\xi=0.4$ are indeed located on intersections of different lines. Based on the experimental knowledge that the $B$-block is preferentially found close to the pore surface, we further concentrate on $\xi=0.4$. For $R=19$ the structure contains perforations, which will not be visible in the experimental imaging procedure. This structure will appear as concentric cylinders $\left(L_{\|}\right)$. For $R=9$, the structure is a mixture of $L_{\|}$and $L_{\perp}$. The standard element of this structure are two opposite small lamellar patches oriented parallel to the pore surface. Along the pore, only the orientation of the elements changes and neighboring elements are orthogonal [see Fig. 12(a)]. The fourfold connections (instead of three) between the elements are short 
a)

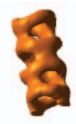

b)

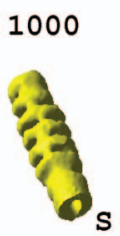

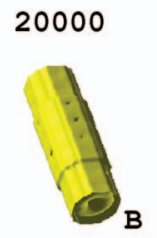

20000

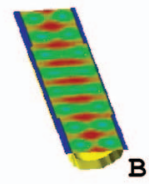

FIG. 12. (Color) (a) High isodensity image of the cagelike structure for $R$ $=9$ and $\xi=0.4$. (b) Structures for the system of Fig. 8 in a rough pore and $\xi=0.4$. The pore radius varies between $R=10$ (center region) and $R=9$ (outer regions). From left to right: Isosurface for the $S$-block (isosurface value $\bar{\theta}_{S}$ ) after 1000 TMS, the same for 20000 TMS, isosurface of the $B$-block (isosurface value $\bar{\theta}_{B}$ ) after 20000 TMS, crop of the isosurface at 20000 TMS combined with an orthogonal slice of the $B$ field in the center of the pore.

and curved cylinders. This mixed structure has interesting features that somewhat resemble the experimental toroidaltype structure of Shin et al. ${ }^{16}$ However, the structures still do not match in all details. Additionally, we varied the small pore radius for fixed $\xi=0.4$ [Fig. 11(c)]. This leads to $L_{\|}$for $R=5,8$ and $L_{\perp}$ for $R=6,7,10$, and 11. In general, we conclude that the decrease of $\chi N$ reliefs some of the stresses present in the stronger phase segregated system, and leads to faster relaxation towards a stable structure.

Finally, the small scale of the experimental nanopores leads to constraints that are not effective in other systems. The results of the previous section show that the structure type can be very sensitive to the pore radius. Small variations of the pore radius (due to roughness) along the pore at relatively larger length scales may therefore affect the structure formation to some extent. This is, in particular, the case in small nanopores, where slightly different pore radii will promote different structure types and connectivity issues (of different types and periodicity) may appear. To study this effect, we prepared a pore with two pore radii, $R=9$ and $10(\xi$ $=0.4$ ), equally distributed along the pore (the thickest part is located in the middle of the pore). Using $L_{0}=8.6$, the bulk characteristic lamellar spacing determined for the system with higher $\chi_{S B}$, the relative pore sizes $R / L_{0}$ vary between 1.05 and 1.16. Keeping in mind that $L_{0}$ will decrease for decreasing $\chi_{S B}$, this range matches the experimental value of 1.3 reasonably well. Following the evolution of this structure [see Fig. 12(b)], we initially observe that simply the stable structures for the individual pore radii are formed and connect ( $L_{\|}$for $R=10$ and the cage structure for $R=9$ ). However, eventually the interface between the different structures acts as a driving force for a transition to another structure. The final and stable structure for the $S$-block is completely perpendicular, with stacked disk for $R=10$, but further away from this region the stacked disks become perforated and adapt the toroidal shape [see Fig. 12(b), second from left]. From a comparison between the experimental image and the simulated 3D structure we observe that cross section of the $B$-block in the center of the pore [Fig. 12(b), utter right] looks very similar to Figs. 1(C) and 1(D) in Ref. 16. Since

the structure for larger $R$ and the same parameters is $L_{\|}$we conclude that this computational system gives a very close match.

\section{Other numerical studies}

The differences in methodology and choices of the parameter range complicate the comparison to results of other computational studies in general. Although most of these studies were not aimed at modeling experimental systems, we note that none of them have reproduced the experimental toroidal-type structure. ${ }^{16}$ Moreover, the dynamics in our method is aimed at following the experimental pathway. Here, we focus on a qualitative comparison to the two most extensive MC studies for symmetric diblock copolymers in nanopores of three different radii: Feng and Ruckenstern ${ }^{23}$ and Chen et $^{\mathrm{al}}{ }^{22}$

In Ref. $23 \chi N$ is relatively low and stacked disks $\left(L_{\perp}\right)$ are found for neutral pores, as expected. The region where $L_{\perp}$ is stable shrinks for increasing pore radius. For large surface interactions, concentric cylinders $\left(L_{\|}\right)$are found. The only alternative structures for intermediate surface fields are $P L\left(R / L_{0} \approx 0.8\right)$ and $L_{\perp, \|}\left(R / L_{0} \approx 1.0\right)$. These results are fully in line with the findings in this article (see Sec. III). Moreover, for $R / L_{0} \approx 1.0$ and an increased $\chi N$, also helices and double helices were obtained for varying surface field strengths. We also find these structures but for somewhat smaller $R$. Previously, we identified these structures as kinetically trapped or stabilized by elongational forces. The particular surface field strength and pore radius at which this effect plays an important role may be expected to depend on the strength of the block-block interface, i.e., the value of $\chi N$. Moreover, also the slight asymmetry of our own block copolymer may lead to a shift of the parameter space where this effect is present.

In Ref. 22, the value of $\chi N$ is considerably higher and the pore radii very small. For small to zero surface fields several structures are found to be stable: Stacked disks $L_{\perp}$, helices $H$, and perforated (concentric) cylinders $P L$. Strangely enough tilted stacked disks are absent, which may have to do with the absence of extensional stresses for the considered pore length. Previously (previous section) we found that for small radii and our choice of $\chi N$ the free energy difference between the helical and perforated lamellar structures can be small. Our results also suggested that the helical structure can be formed via the merging of perforations in the perforated structure, and subsequently that $P L$ is an intermediate structure in the $D \rightarrow P L \rightarrow H$ evolution. The fact that here $P L$ is found for small surface fields is therefore not completely unexpected. For large surface fields, $L_{\|}$is stable. The only true discrepancy between this study and ours is the stacked circle structure found for $D=26$ (Ref. 22) close to the stability region of the $L_{\|}$structure. This structure, similar to $L_{\|}$but with short bounded instead of an infinite cylinder in the pore center, could originate from packing problems in combination with a relatively large surface field. Although the bulk distance $L_{0}$ was not determined explicitly, we can take $L_{0}=16$ from counting the number of stacked disks in the cylinder, and find $R / L_{0}=0.81$. We refrain from further specu- 
lations about the origin of this structure, and conclude that it is either an exotic structure or due to the higher $\chi N$.

Finally, we briefly consider the results of a nanopore study that employed dissipative particle dynamics (DPD). In Ref. 41 a $A_{5} B_{5}$ block copolymer system is simulated, both in neutral pores and pores with surface fields. The pore wall is not included as a geometrical constraint, but as soft core DPD beads with strong repulsion. The effect of extensional forces is not considered. The authors find that $L_{\perp}$ always forms in neutral pores, except for the smallest pore radius considered $(R=7)$ (Ref. 41) where a double helix $D H$ was found. For pores with strong surface fields only $L_{\|}$forms, except for the smallest radius where $L_{\perp}$ is obtained. For surface field strength between these two extrema, several structures are obtained and hopping between different states of the system is observed. Although not mentioned as such, the structures in Fig. 5 of (Ref. 41) show that they can be arrested in alternative morphologies. In particular, helical structures were found to serve as intermediates. Although the parameter range in this study is different and limited, it confirms our findings that the system can rather easily adapt to incommensurablity in larger nanopores. Moreover, for the intermediate parameter range (small, nonzero surface fields) there is a delicate balance between entropic and energetic contributions to the free energy, and structural transitions are mediated by helicity. In our more systematic study we show that structures can sometimes be arrested in these transition (helical) states. Moreover, we showed that for very small pore radii perpendicular structures can be found for relatively large surface fields.

\section{CONCLUSIONS}

We have used a DDFT method to study the behavior of a slightly asymmetric diblock copolymer $S_{10} B_{12}$ confined in a cylindrical nanopore. This block copolymer forms lamellae in the bulk, and was chosen to model the experimental system in Ref. 17. We find many structures that were also identified in nanopore studies for completely symmetric diblock copolymers, but also a few new structures. However, almost all exotic structures are metastable. Our aim here is to identify general rules for structure formation in nanopores. We have focused on the intermediate surface strength in the parameter space, where the effective surface field, determined by the difference of the two block-surface interactions $\xi$, is relatively small. Outside this region, curved concentric cylinders (in analogy with the thin film notation denoted by $L_{\|}$) are stable based on energetic arguments. The sign of the surface field determines the block that is preferentially found to wet the pore surface: For positive $\xi$ the $B$-block is found close to the pore surface, and for negative $\xi$ the $S$-block is preferential close to this surface. Moreover, for a small and vanishing surface field the elastic chain deformations present in the concentric cylinders are avoided by the formation of a stacked disk structure (denoted as $L_{\perp}$ ). The transition point from parallel structures $\left(L_{\|}\right)$to perpendicular structures $\left(L_{\perp}\right)$ is located somewhere inside this region, and we find many alternative structures. Analysis of the free energy and the determination of a phase diagram of equilibrium structures shows that all of these structures except $L_{\|}, P L$, and $L_{\perp}$ are metastable, and we have considered possible causes in detail. For stronger surface fields, that in general favor surface reconstructions (parallel structures), commensurability of the pore radius $R$ and the natural lamellar domain distance $L_{0}$ is important to avoid large compression or extension of the chains in parallel structures. For weaker surface fields, favoring perpendicular structures, commensurability of the pore length $L_{x}$, and the natural lamellar domain distance $L_{0}$ is important to avoid large extensional forces. The commensurability issues, together with sometimes extremely slow transition dynamics, were found to cause the formation of alternative structures.

For stronger surface fields, phase separation is initiated by the presence of the interacting pore surface. As a result, the pathway from the homogeneous melt to more stable alternative structures includes a first stage of parallel structures or surface reconstructions, even when $L_{\|}$is not equilibrium. Subsequently, when the driving force is relatively large, holes are formed (for larger $R$, due to undulations that form connections between different layers) and connect: A helical structure is formed at a larger scale. The most probable origin of this helicity is that helical structures can more easily accommodate structural reformations at a higher level by adapting their spacing and pitch than defected $L_{\perp}$ structures. At the final stages, the helical structures line up by defect movement: The formation and destruction of threefolded connections. We find that these helical structures cost few energy to the system. Along this pathway, the structure can get kinetically trapped depending on the frustration that it experiences. We find metastable $L_{\|}$, structures with holes (denoted by $P L$, and similar to the mesh or catenoid structures in Refs. 23 and 22, respectively), helices, or double helices for decreasing surface field strength. In general, the transformation of the intermediate structures to other structures can be slowed down, or even be halted, by the presence of extensional forces due to incommensurability along the pore.

For weaker surface fields the influence of the confinement on the initial stages is less substantial. As a result the initial structure is very interconnected and defected. The mechanism for the removal of these defects, however, is very similar to the one described above: Small helical domains and threefold connections play an important role. Here, the extensional forces play a major role, and determine whether stacked disks, tilted stacked disks, or helical-like structures (which are actually clusters of tilted stacked disks under opposite angles, connected via helical domains) are formed.

We have also considered the experimental system of Shin et al. ${ }^{16}$ in which a new toroidal-type structure was observed. Our results suggest that for weaker surface fields and small pore radius the structures are very sensitive to pore radius variations. Moreover, these pore radius variations give rise to interfaces between the stable structures for constant pore radii that exist in parts of the pore. These interfaces act as the driving force for the transition to other structures. For a certain surface field strength $(\xi=0.4)$ and constant pore radius, we find a cagelike structure for a pore radius close to the experimental value, and perforated concentric cylinders (that appear as concentric cylinders in the experimental im- 
aging procedure) for large pore radii. Moreover, by including the roughness of the experimental pore into our calculation in the form of a step profile, we were able to obtain a $3 \mathrm{D}$ structure that is very similar to the experimental structure.

\section{ACKNOWLEDGMENTS}

We acknowledge support of Stichting Nationale Computer Faciliteiten. We thank Thomas Russell for pointing out the application of pores in nanotechnology, and Jan van Male for useful discussions.

${ }^{1}$ Q. Wang, in Nanostructured Soft Matter: Experiment, Theory, Simulation and Perspectives, edited by A. V. Zvelindovsky (Springer, Dordrecht, 2007), pp. 498-528.

${ }^{2}$ F. S. Bates and G. H. Fredrickson, Phys. Today 52(2), 32 (1999).

${ }^{3}$ M. W. Matsen, Curr. Opin. Colloid Interface Sci. 3, 40 (1998).

${ }^{4}$ K. Binder, Adv. Polym. Sci. 138, 1 (1999).

${ }^{5}$ M. J. Fasolka and A. M. Mayes, Annu. Rev. Mater. Res. 31, 323 (2001).

${ }^{6}$ T. Hashimoto, in Nanostructured Soft Matter: Experiment, Theory, Simulation and Perspectives, edited by A. V. Zvelindovsky (Springer, Dordrecht, 2007), pp. 45-98.

${ }^{7}$ L. H. Radzilowski, B. L. Carvalho, and E. L. Thomas, J. Polym. Sci., Part B: Polym. Phys. 34, 3081 (1996).

${ }^{8}$ H. P. Huinink, J. C. M. Brokken-Zijp, M. A. van Dijk, and G. J. A. Sevink, J. Chem. Phys. 112, 2452 (2000).

${ }^{9}$ Q. Wang, P. F. Nealy, and J. J. de Pablo, Macromolecules 34, 3458 (2001).

${ }^{10}$ A. Knoll, K. S. Lyakhova, A. Horvat, G. Krausch, G. J. A. Sevink, A. V. Zvelindovsky, and R. Magerle, Nat. Mater. 3, 886 (2004).

${ }^{11}$ T. Xu, A. V. Zvelindovsky, G. J. A. Sevink, K. S. Lyakhova, H. Jinnai, and T. P. Russell, Macromolecules 38, 10788 (2005).

${ }^{12}$ X. He, M. Song, H. Liang, and C. Pan, J. Chem. Phys. 114, 10510 (2001).

${ }^{13}$ G. J. A. Sevink, A. V. Zvelindovsky, J. G. E. M. Fraaije, and H. P. Huinink, J. Chem. Phys. 115, 8226 (2001).

${ }^{14}$ A. Knoll, A. Horvat, K. S. Lyakhova, G. Krausch, G. J. A. Sevink, A. V. Zvelindovsky, and R. Magerle, Phys. Rev. Lett. 89, 035501 (2002).

${ }^{15}$ M. J. Fasolka, P. Banerjee, A. M. Mayes, G. Pickett, and A. C. Balazs, Macromolecules 33, 5702 (2000).

${ }^{16}$ K. Shin, H. Q. Xiang, S. I. Moon, T. Kim, T. J. McCarthy, and T. P. Russell, Science 306, 76 (2004).
${ }^{17}$ H. Q. Xiang, K. Shin, T. Kim, S. I. Moon, T. J. McCarthy, and T. P. Russell, Macromolecules 37, 5660 (2004).

${ }^{18}$ Y. Y. Wu, G. S. Cheng, K. Katsov, S. W. Sides, J. F. Wang, J. Tang, G. H. Fredrickson, M. Moskovits, and G. D. Stucky, Nat. Mater. 3, 816 (2004).

${ }^{19}$ H. Q. Xiang, K. Shin, T. Kim, S. I. Moon, T. J. McCarthy, and T. P. Russell, J. Polym. Sci., Part B: Polym. Phys. 43, 3377 (2005).

${ }^{20}$ Y. Sun, M. Steinhart, D. Zschech, R. Adhikari, G. H. Michler, and U. Gösele, Macromol. Rapid Commun. 26, 369 (2005).

${ }^{21}$ W. Li, R. A. Wickham, and R. A. Garbary, Macromolecules 39, 806 (2006).

${ }^{22}$ P. Chen, X. He, and H. Liang, J. Chem. Phys. 124, 104906 (2006).

${ }^{23}$ J. Feng and E. Ruckenstein, Macromolecules 39, 4899 (2006).

${ }^{24}$ J. Feng and E. Ruckenstein, J. Chem. Phys. 125, 164911 (2006).

${ }^{25}$ Q. Wang, J. Chem. Phys. 126, 024903 (2007).

${ }^{26}$ I. W. Hamley, The Physics of Block Copolymers (Oxford University Press, Oxford, 1998).

${ }^{27}$ J. G. E. M. Fraaije, B. A. C. van Vlimmeren, N. M. Maurits, M. Postma, O. A. Evers, C. Hoffmann, P. Altevogt, and G. Goldbeck-Wood, J. Chem. Phys. 106, 4260 (1996).

${ }^{28}$ B. A. C. van Vlimmeren, N. M. Maurits, A. V. Zvelindovsky, G. J. A. Sevink, and J. G. E. M. Fraaije, Macromolecules 32, 646 (1999).

${ }^{29}$ M. W. Matsen, M. Schick, Phys. Rev. Lett. 72, 2660 (1994).

${ }^{30}$ G. J. A. Sevink, A. V. Zvelindovsky, B. A. C. van Vlimmeren, N. M. Maurits, and J. G. E. M. Fraaije, J. Chem. Phys. 110, 2250 (1999).

${ }^{31}$ N. M. Maurits, J. G. E. M. Fraaije, P. Altevogt, and O. A. Evers, Comput. Theor. Polym. Sci. 6, 1 (1996).

${ }^{32}$ N. M. Maurits and J. G. E. M. Fraaije, J. Chem. Phys. 107, 5879 (1997).

${ }^{33}$ J. J. Freire and C. McBride, Macromol. Theory Simul. 12, 237 (2003).

${ }^{34}$ G. Wang, G. Yan, P. F. Naeley, and J. J. de Pablo, J. Chem. Phys. 112, 450 (2000).

${ }^{35}$ H. Morita, T. Kawakatsu, M. Doi, D. Yamaguchi, M. Takenaka, and T. Hashimoto, J. Phys. Soc. Jpn. 73, 1371 (2004).

${ }^{36}$ H. P. Huinink, J. C. M. Brokken-Zijp, M. A. van Dijk, and G. J. A. Sevink, Macromolecules 34, 5325 (2001).

${ }^{37}$ K. S. Lyakhova, A. V. Zvelindovsky, and G. J. A. Sevink, Macromolecules 39, 3024 (2006).

${ }^{38}$ M. L. Ma, V. Krikorian, J. H. Yu, E. L. Thomas, and G. C. Rutledge, Nano Lett. 6, 2969 (2006).

${ }^{39}$ A. V. Kyrylyuk and J. G. E. M. Fraaije, J. Chem. Phys. 125, 164716 (2006).

${ }^{40}$ J. N. Owens, I. S. Gancarz, J. T. Koberstein, and T. P. Russell, Macromolecules 22, 3380 (1989).

${ }^{41}$ J. Feng, H. Liu, and Y. Hu, Macromol. Theory Simul. 15, 674 (2006). 